\title{
Measurement and modeling of electron-cloud-induced betatron tune shifts at the Cornell Electron-Positron Storage Ring test accelerator
}

\author{
S. Poprocki, ${ }^{*}$ S. W. Buechele, J. A. Crittenden, K. Rowan, D. L. Rubin, and J. E. San Soucie \\ CLASSE, Cornell University, Ithaca, New York 14853, USA
}

(Received 1 January 2019; published 1 August 2019)

\begin{abstract}
We report on extensive measurements at the Cornell Electron-Positron Storage Ring of electron-cloudinduced betatron tune shifts for trains of positron bunches at 2.1 and $5.3 \mathrm{GeV}$ with bunch populations ranging between $0.64 \times 10^{10}$ and $9.6 \times 10^{10}$. Measurements using a witness bunch with variable distance from the end of the train and variable bunch population provide information on cloud decay and cloud pinching during the bunch passage. We employ Monte Carlo simulations of the reflection and absorption of synchrotron radiation photons to determine the pattern of absorption sites around the circumference of the storage ring. The GEANT4 simulation toolkit is used to model the interactions of the photons with the beampipe wall and determine the production energy and location distributions of the photoelectrons which seed the electron cloud. An electron cloud buildup model based on fitted ring-averaged secondary-yield properties of the vacuum chamber predicts tune shifts in good agreement with the measurements.
\end{abstract}

DOI: 10.1103/PhysRevAccelBeams.22.081001

\section{INTRODUCTION}

The buildup of low-energy electron densities in the vacuum chamber of a positron storage ring can result in betatron tune shifts, instabilities and emittance growth. We describe techniques to measure electron-cloud-induced tune shifts, and to use the measurements to constrain predictive numerical models of electron cloud phenomena. Analytic and numerical treatments of electron cloud (EC) contributions to coherent tune shifts were originally presented in Ref. [1] and further developed in Ref. [2].

The Cornell Electron-Positron Storage Ring (CESR) was reconfigured as a test accelerator in 2008 [3]. A comprehensive summary of the project, which included electroncloud buildup and low-emittance lattice studies, can be found in the CESRTA Phase I Report [4]. The results reported here concern three lattice configurations of the CESR ring: the test accelerator configurations at $2.1 \mathrm{GeV}$ and at $5.3 \mathrm{GeV}$, and the $6.0 \mathrm{GeV}$ upgrade to be commissioned in 2019. Table I lists the parameters of these three lattice configurations.

In Sec. II we discuss and compare methods of measuring bunch-by-bunch betatron tune shifts. A comprehensive set of measurements along trains of positron bunches at 2.1 and $5.3 \mathrm{GeV}$ is shown. We describe in Sec. III the full

\footnotetext{
*stp44@cornell.edu
}

Published by the American Physical Society under the terms of the Creative Commons Attribution 4.0 International license. Further distribution of this work must maintain attribution to the author(s) and the published article's title, journal citation, and DOI. procedure of electron cloud simulation starting with the generation of photons from synchrotron radiation, tracking of the photons in a 3D model of the vacuum chamber including reflections and absorption of the photons, the production of photoelectrons, the buildup of electron densities along a train of bunches, and the calculation of betatron tune shifts.

Although electron cloud buildup models have been successful in simulating tune shifts [5-8] and vertical

TABLE I. Lattice and beam parameters for the three configurations of the CESR ring addressed in this report: the 2.1 and $5.3 \mathrm{GeV}$ lattice configurations for which betatron tune shifts were measured and for which simulations were performed, and the 6.0 GeV configuration for which the model was used to assess effects of electron cloud buildup on performance.

\begin{tabular}{lccc}
\hline \hline Beam energy (GeV) & 2.085 & 5.289 & 6.000 \\
\hline Circumference (m) & \multicolumn{3}{c}{768.44} \\
Bunch current (mA/bunch) & $0.4-0.7$ & $2.0-6.0$ & $2.2-4.4$ \\
Number of bunches & 30 & 20 & $45-90$ \\
Beam current (mA) & $12-21$ & $40-120$ & 200 \\
rf frequency (MHz) & & 500 & \\
Energy loss per turn (MeV) & 0.19 & 1.1 & 1.8 \\
Momentum compaction $\left(10^{-3}\right)$ & 6.7 & 9.2 & 5.7 \\
Bunch length (mm) & 9.2 & 15.8 & 15.6 \\
Bunch spacing (ns) & 14 & 14 & 14 \\
Energy spread (10 & \\
Horizontal tune & 8.1 & 6.5 & 7.6 \\
Vertical tune & 14.5639 & 11.2853 & 16.545 \\
Synchrotron tune & 9.5984 & 8.7914 & 12.63 \\
Horizontal emittance (nm) & 0.07354 & 0.04623 & 0.03416 \\
Vertical emittance (nm) & 3.2 & 97 & 30 \\
\hline \hline
\end{tabular}


emittance growth $[9,10]$ in general agreement with measurements, their predictive power has been limited. Furthermore, no model has yet reproduced measurements of horizontal and vertical tune shifts over as wide a range of bunch population and beam energy as considered in this analysis.

Models of electron cloud formation, which are the basis for prediction of tune shifts and emittance growth, typically depend on phenomenological descriptions of much of the underlying physics. The model parameters are tuned so that simulations based on the model are consistent with measurements. In an effort to improve the predictive power of the model, we replace the phenomenological descriptions with first-principles calculations for two of the processes critical to the determination of cloud growth. We employ the SYNRAD3D [11] code to calculate azimuthal location distributions of photons absorbed on the vacuum chamber walls, including their energies and angles of incidence throughout the circumference of the CESR ring. These calculations include photon reflectivity and the effect of surface roughness. The GEANT4 [12] simulation tookit is then used to calculate the emission of photoelectrons by the absorbed photons into the vacuum chamber volume [13]. The description of the physics of secondary-electron yield (SEY) remains phenomenological. Parameters of the secondary yield model are fit to the large dataset of betatron tune shift measurements collected at CESR.

Section IV discusses these results and draws conclusions about how betatron tunes respond to various cloud buildup characteristics. Finally, the model with the best-fit SEY parameters is applied to obtain estimates for the consequences of electron cloud buildup for operation of the major CESR upgrade (CHESS-U) to be completed in 2019 [14].

\section{TUNE SHIFT MEASUREMENT}

Tune shifts have been measured in a number of ways at CESRTA. A relatively straightforward technique is to kick the entire train all at once with a single-turn pinger, and then record turn-by-turn position data for each bunch. In the limit where the positron bunch is oscillating transversely on passage through a static electron cloud, an FFT of the position data yields the betatron tune $[4,5]$ and the shift due to the presence of the cloud. But because the cloud follows the pinger-induced horizontal motion of the train, the measurement of horizontal tune shifts by this method is difficult to interpret. In general, low-energy electrons emitted from the top (bottom) of the vacuum chamber are accelerated by the positron bunch and strike the bottom (top) of the chamber. In the dipole magnets, the resulting secondaries are trapped by the magnetic field lines in a vertical band of width comparable to that of the bunch. A horizontal ping, with pulse length long compared to the train length, moves the bunch train coherently, and thus the cloud as well. This measurement technique is thus insensitive to horizontal tune shifts, since the test bunch receives no coherent kick from the comoving cloud. A further limitation of the technique is that the presence of multiple peaks in the FFT from coupled-bunch motion contaminates the signal.

Better results are obtained by enabling the bunch-bybunch feedback, and disabling it one bunch at a time to measure the tune of that bunch. The self-excitation (no external kick applied) typically yields a measurable signal, but the precision of the measurement is improved by kicking the single bunch with a gated strip-line kicker. This technique is further refined by driving the bunch with a tune tracker [15]. The tune tracker phase-locks an oscillator to the observed betatron signal, providing a frequency source for coherent excitation of steady-state betatron motion. It also provides a digital clock that is synchronized to the instantaneous betatron motion, which can be used for synchronous detection of betatron signals. The measurements with the tune tracker were done separately in horizontal and vertical planes. Betatron tunes were measured along the train, one bunch at a time, and bunch currents were monitored and topped off between measurements as needed-typically after every $1-5$ bunches.

Tune shifts measured using the pinging method for 20-bunch trains of positrons at $5.3 \mathrm{GeV}$ and for several values of the bunch current are shown in Fig. 1. The bunch spacing is $14 \mathrm{~ns}$. For the CESR revolution frequency of $390 \mathrm{kHz}$, a tune shift of $1 \mathrm{kHz}$ corresponds to a fractional tune shift $\Delta Q=0.0026$. Large bunch-to-bunch fluctuations as well as overlap of data are observed. The tune shift measurements obtained using the tune tracker are shown in Fig. 2, and exhibit vertical tune shifts increasing monotonically with bunch current. These measurements are more useful than those obtained via the pinging method since (a) the single bunch tune measurement using the tune

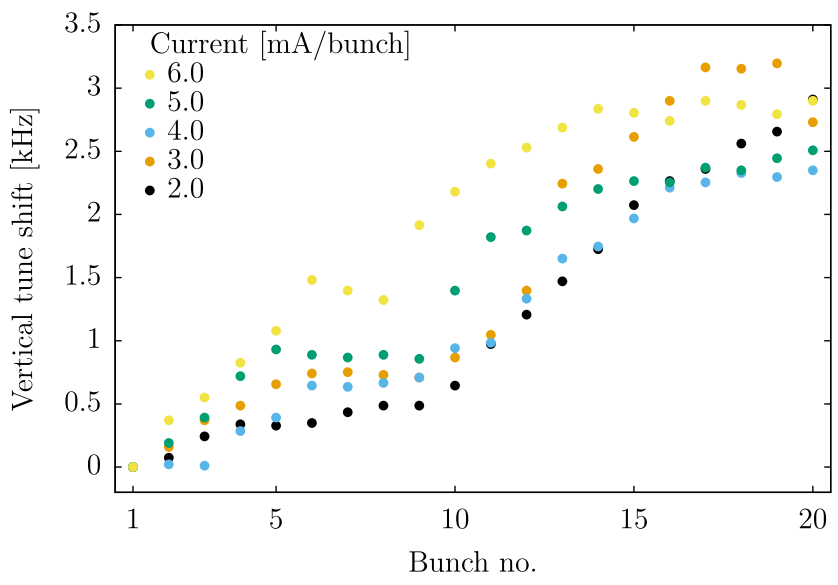

FIG. 1. Vertical betatron tune shifts measured using the "pinging" method along a 20-bunch train of positrons at 5.3 GeV for values of the bunch current ranging from 2 to $6 \mathrm{~mA} /$ bunch $\left(3.2-9.6 \times 10^{10}\right.$ bunch populations $)$. 

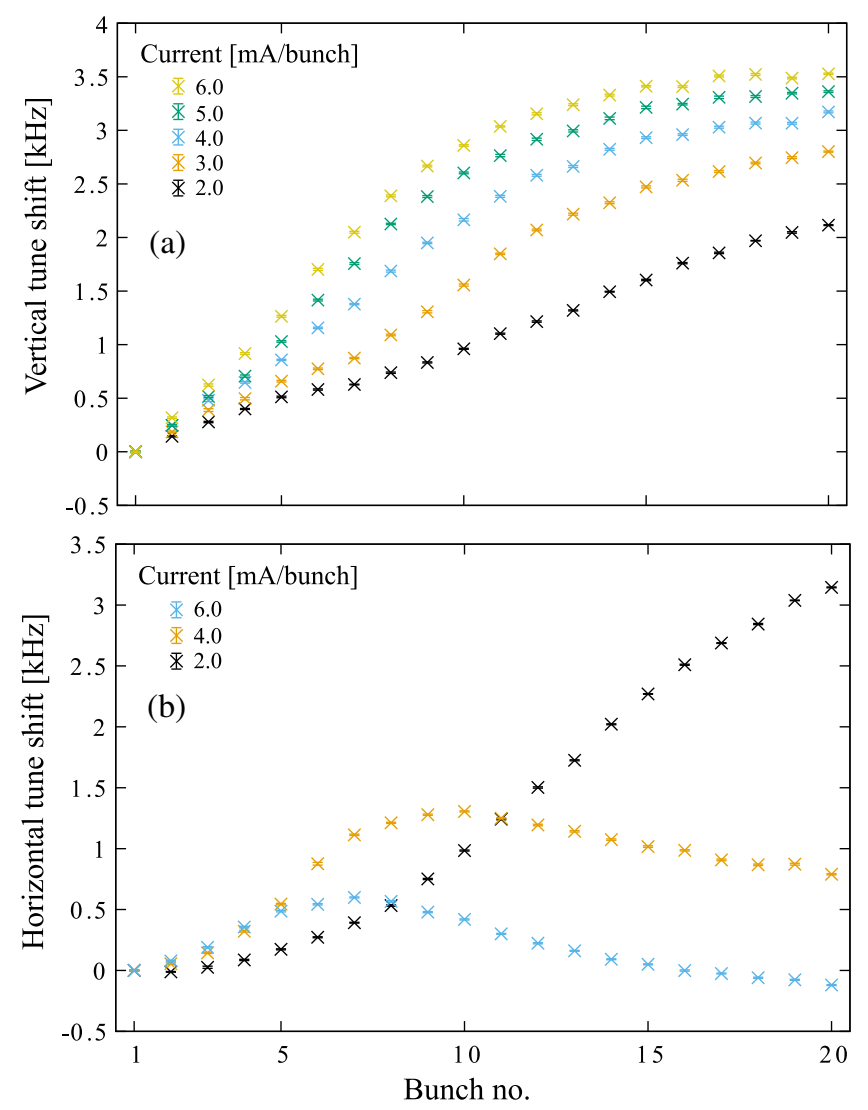

FIG. 2. Tune shifts measured in the (a) vertical and (b) horizontal planes using the tune tracker for a 20-bunch train of positrons with values for the bunch current ranging between 2 and $6 \mathrm{~mA} /$ bunch $\left(3.2-9.6 \times 10^{10}\right.$ bunch populations $)$ at $5.3 \mathrm{GeV}$. Data were recorded separately for each of the two planes.

tracker is more accurate, and (b) all of the other bunches in the train are stabilized via feedback, thus eliminating coupled bunch motion.

The horizontal tune shift reaches a maximum along the train, decreasing for later bunches. The maximum tune shift occurs earlier in the train as bunch current increases. This behavior is understood in terms of the "cloud splitting" effect in dipole magnetic fields. The cloud electron energies resulting from the attractive kick imparted by passage of the positron bunch increase with bunch current. The electron trajectories are pinned along the vertical field lines in a tight spiraling motion. The secondary-emission yield has a strong dependence on the incident electron energy, with peak yield at an energy of a few hundred eV. (For a description of secondary-electron emission processes, see, e.g., Ref. [16].) The electrons near the positron bunch in the vertical plane containing the beam are accelerated to the highest energies, so these are the first to strike the vacuum chamber wall at energies exceeding the maximum in the SEY energy dependence. As the cloud builds up during the passage of the train, the location on the beampipe wall of maximum average SEY moves away from the vertical
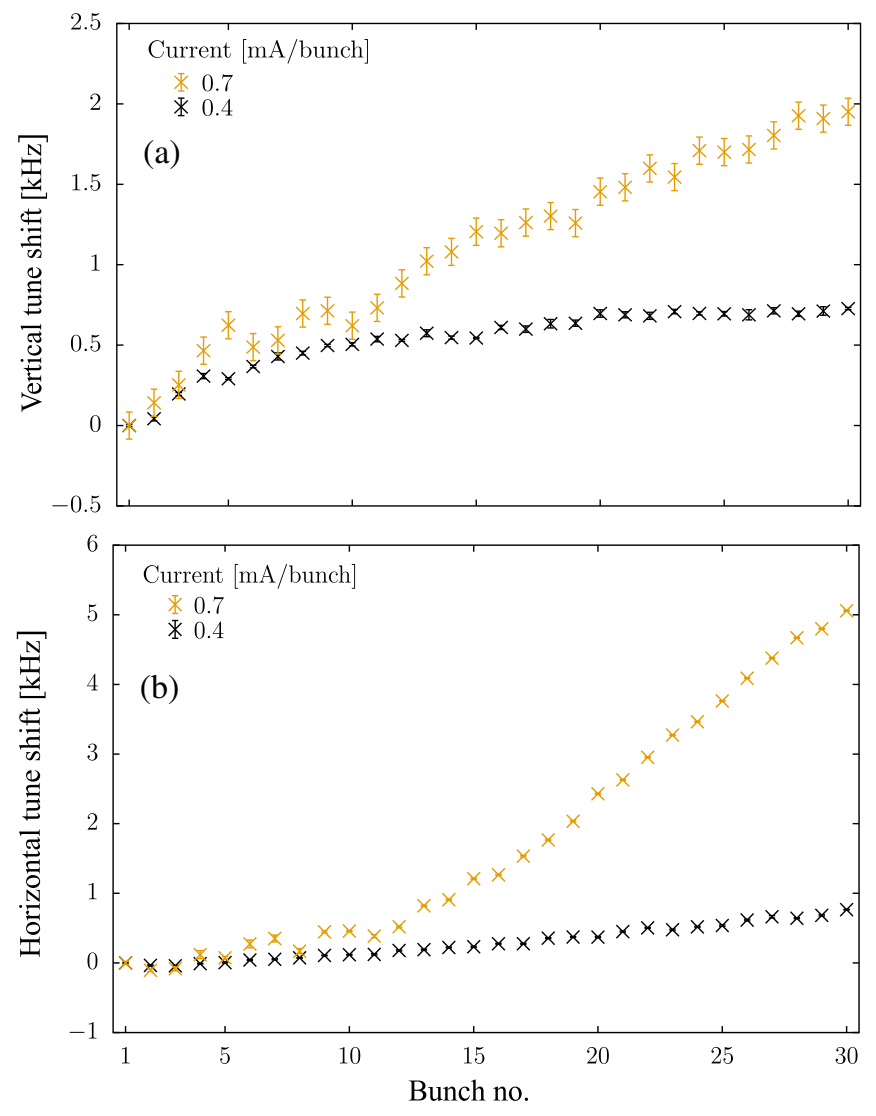

FIG. 3. Tune shifts measured in the (a) vertical and (b) horizontal planes using the tune tracker for a 30-bunch train of positrons at 0.4 and $0.7 \mathrm{~mA} /$ bunch $\left(0.64 \times 10^{10}\right.$ and $1.12 \times 10^{10}$ bunch populations) at $2.1 \mathrm{GeV}$.

plane containing the beam and the dense vertical stripe of the cloud first widens and then splits into two stripes.

Tune shift measurements taken with the tune tracker for positrons at $2.1 \mathrm{GeV}$ are shown in Fig. 3. The fluctuations and larger uncertainties observed in the vertical tune shift measurements at $0.7 \mathrm{~mA} /$ bunch were reduced in subsequent measurements at the other bunch currents by averaging over measurements collected at an increased acquisition rate. The horizontal tune shift depends on the bunch current in a nonlinear way, increasing by more than a factor of 5 as the bunch current increases from 0.4 to $0.7 \mathrm{~mA} /$ bunch. The roughly linear increase in tune shift with bunch number, beginning with bunch 11, is shown by the modeling to be characteristic of the cloud growth in the dipoles (see Sec. IV). Note the very different bunch currents in the measurements at 2.1 and $5.3 \mathrm{GeV}$ and the nonlinear dependence of tune shift on bunch current and beam energy. While the synchrotron photon emission rate increases linearly with beam energy and bunch current, the higher beam kicks result in cloud electron energy distributions which span the maximum in the dependence of SEY on incident electron energy, leading to saturation. 


\section{SIMULATION METHOD}

The modeling of electron cloud effects on beam dynamics proceeds in four steps: (1) 3D calculation of the pattern of absorbed synchrotron radiation around the ring including the effects of photon reflections [11], (2) simulation of the interactions of absorbed photons with the vacuum chamber wall which lead to the emission of electrons $[12,17,18]$, (3) a time-sliced weak-strong model $[19,20]$ for electron cloud development along a train of positron bunches, including a phenomenological model for SEY from the beampipe walls, and (4) calculations of betatron tune shifts using the space-charge electric field gradients derived from the cloud buildup model [4-6,21]. The physics of SEY was parametrized as described in Ref. [22] and the parameters were fit to the tune shift measurements using an iterative optimization procedure. Note that the SEY parameters are the only free parameters in the simulation. These four steps are described in Secs. III A-III D below.

\section{A. Synchrotron radiation photon tracking}

An essential tool in this study is the photon-tracking code SYNRAD3D [11], which simulates the generation of individual photons radiated by the positron beam, and incorporates a user-defined 3D model of the vacuum chamber to model the reflection and absorption of photons using the BMAD library [23] and x-ray data from an LBNL database [24]. Figure 4 shows a plan view of photon trajectories in a region of the CESR ring which includes $\mathrm{x}$-ray beam line exit windows at which incident photons are not included in the tally of photons absorbed in the vacuum chamber walls.

Photon reflectivity plays a crucial role in electron cloud buildup, since it determines the distribution of photon absorption sites around the ring. Furthermore, without

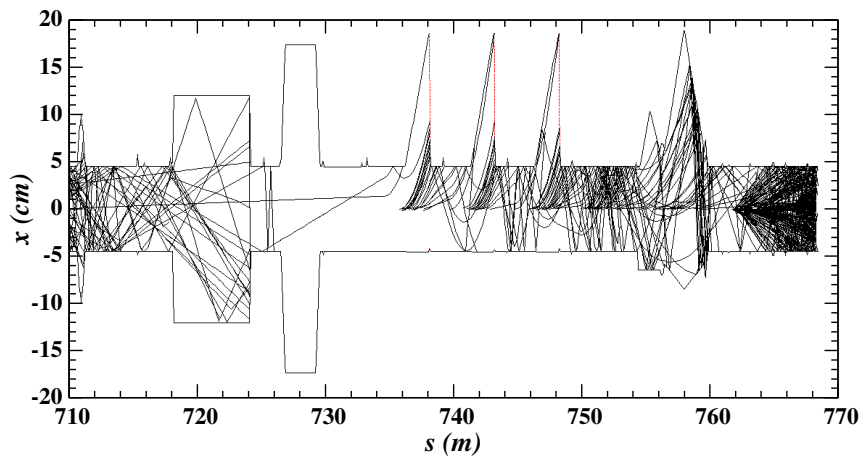

FIG. 4. Modeled photon trajectories in a section of the CESR ring which includes various vacuum system components as well as X-ray beam exit ports. Projections of the horizontal position $x$ of the photons relative to the positron reference orbit are shown. The positrons travel in the direction of increasing $s$ coordinate. The red vertical lines represent the exit port windows; any photon hitting those surfaces is terminated and excluded from the absorbed-photon rate. photon reflectivity, few photons could be absorbed on the top and bottom of the beampipe, where photoelectron production is the primary source of cloud generation in the vertical plane containing the beam, which is particularly important in dipole magnetic fields.

A microgroove structure on the surface of the CESR vacuum chamber has been measured using atomic force microscopy and studied in x-ray beams [25]. These grooves are roughly parallel to the beam axis and are understood to be caused by the beampipe extrusion process. Their effect is accounted for by incorporating the groove structure into the beampipe model and simulating specular reflections in the grooves. Figure 5 shows a diagram of the modeled grooves used in the photon-tracking simulation, and Fig. 6 shows the effect of the grooves on the photon tracks.

The transverse absorption location distribution in Fig. 7 shows the consequence of the larger reflection angles from grooves in the dipole regions for the case of the $5.3 \mathrm{GeV}$ beam. The absorbed photon rate on the top and bottom of the beampipe increases by a factor of about 3 when the grooves are included.

The reflectivity is also critically dependent on the material composition of the vacuum chamber wall. Figure 8 shows the fraction of photons reflected as a function of photon energy for a $5^{\circ}$ grazing angle for aluminum with and without $\mathrm{C}$ and $\mathrm{CO}$ surface layers. The data were obtained from the LBNL database [24]. In validating our modeling studies, we have chosen to use the 5-nm CO layer, as motivated in Ref. [25].

The photon tracking simulation identifies $10^{6}$ locations around the CESR ring where photons are absorbed, along with the energy and incident angle of the photon. All of the simulation results shown below assume the microgroove structure, a surface roughness parameter of 100-nm rms for the diffuse component of the scattering, and a 5-nm CO surface layer. The surface roughness parameter value was derived from the measurements described in Ref. [25].

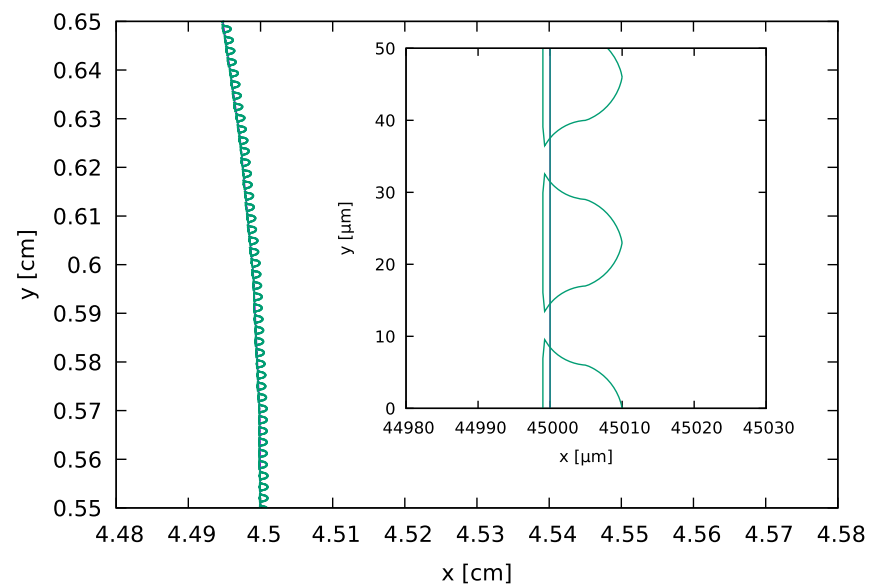

FIG. 5. Schematic diagram of the 10- $\mu$ m-deep grooves on the CESR vacuum chamber wall used in the photon reflectivity model. The simulated vacuum chamber is the union of geometric shapes. 

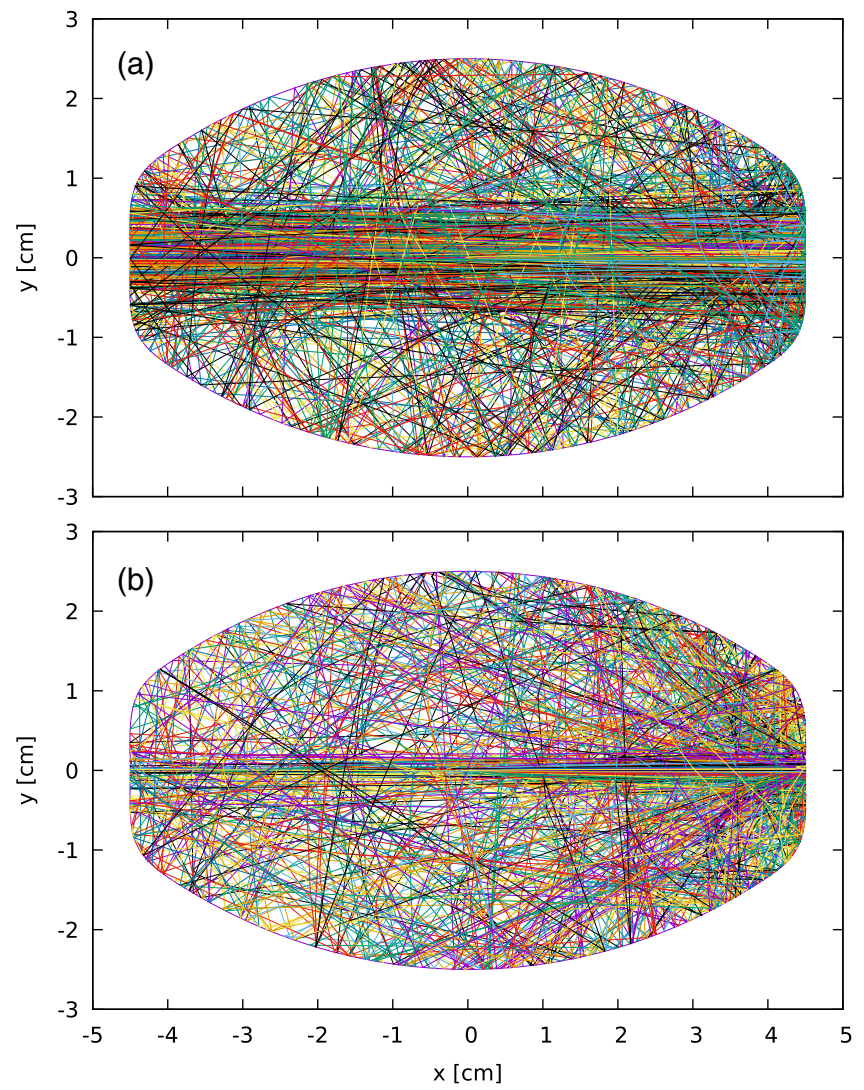

FIG. 6. Examples of photon trajectories (a) without the groove pattern on the vacuum chamber wall, and (b) with grooves. The groove pattern results in significantly enhanced scattering out of the horizontal midplane. The apparent curvature in the tracks is a consequence of the longitudinal bend in the reference trajectory in the dipole. (Beam energy is $5.3 \mathrm{GeV}$.)

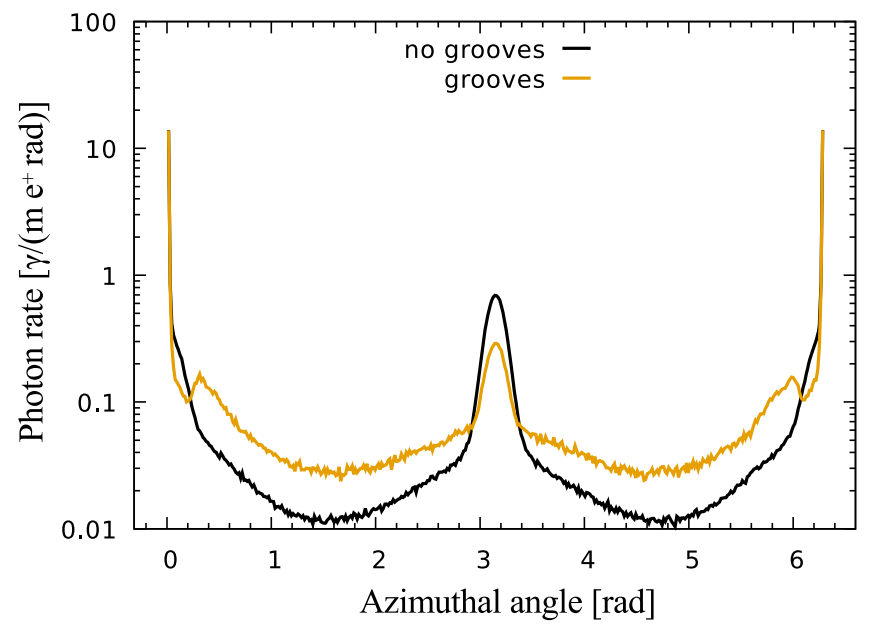

FIG. 7. Comparison of the azimuthal absorption location of the absorbed photons in the dipole regions when microgrooves are introduced in the CESR vacuum chamber geometry. The azimuthal angle is defined to be $180^{\circ}$ in the horizontal plane containing the beam axis on the inside of the ring. (Beam energy is $5.3 \mathrm{GeV}$.)

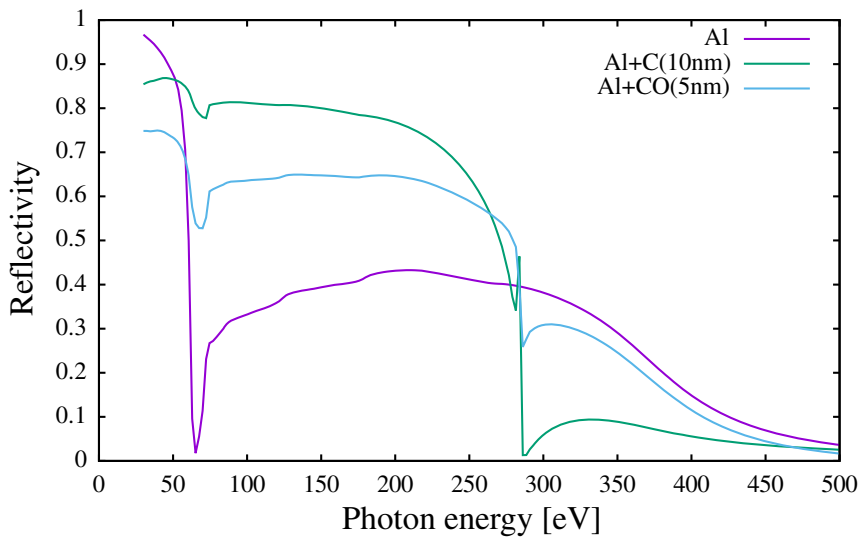

FIG. 8. Smooth-surface photon reflectivity versus photon energy for aluminum, aluminum with a 10-nm carbon layer, and aluminum with a 5-nm carbon monoxide layer, for photons incident at a $5^{\circ}$ grazing angle.

Figure 9(a) shows the linear density per beam particle of absorption sites around the 768-m-circumference CESR ring. The higher densities near the former collider interaction regions at $s=0, s=384 \mathrm{~m}$, and $s=768 \mathrm{~m}$ result from the higher-strength dipole magnets outboard of the
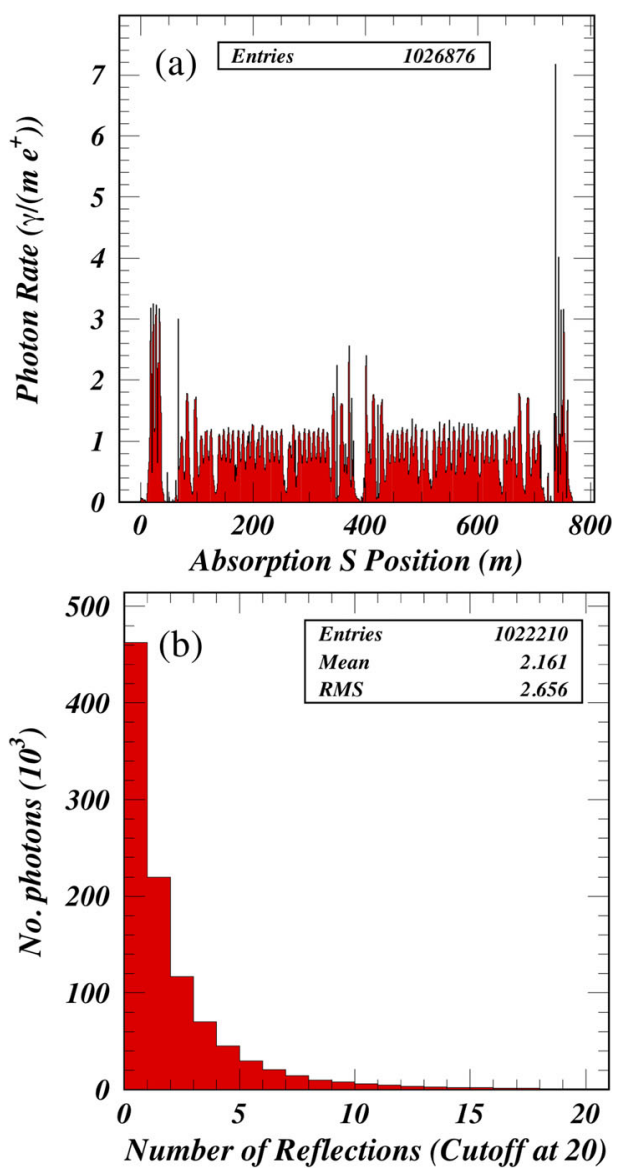

FIG. 9. Distributions of absorbed photons in (a) absorption location along the CESR ring, and (b) number of prior reflections. (Beam energy is $5.3 \mathrm{GeV}$.) 


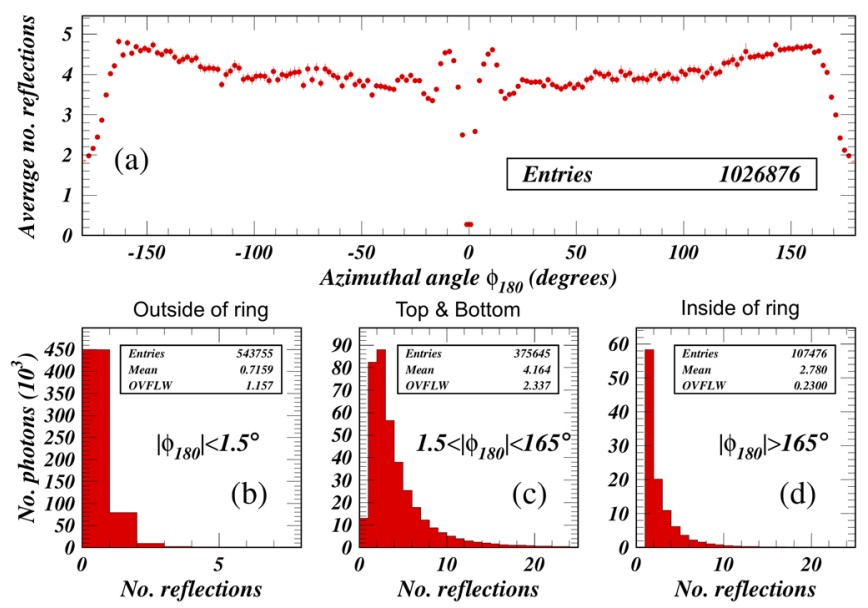

FIG. 10. Average number of prior reflections for absorbed photons summed over (a) the full ring as a function of the azimuthal absorption location on the vacuum chamber wall, $\Phi_{180}$. The distributions in the number of reflections are shown for the three azimuthal regions (b) $\left|\Phi_{180}\right|<1.5^{\circ}$, (c) $1.5^{\circ}<\left|\Phi_{180}\right|<165^{\circ}$, and (d) $\left|\Phi_{180}\right|>165^{\circ}$. (Beam energy is $5.3 \mathrm{GeV}$.)

straight sections where detectors were formerly installed. The distribution in the number of reflections prior to absorption is shown in Fig. 9(b). About half of the absorbed photons are absorbed on the first wall strike. The photon energy is conserved in the modeled reflection process. However, since the reflection probability is a strong function of the incident photon energy, the photons absorbed after undergoing a prior reflection are generally of lower energy than those absorbed without prior reflections.

Only reflected photons strike the top, bottom and inner walls of the vacuum chamber. The typical number of reflections before absorption depends on the azimuthal angle $\Phi_{180}$ of the absorption site location, where $\Phi_{180}$ ranges from $-180^{\circ}$ to $+180^{\circ}$ with its origin in the midplane on the outside of the ring. Figure 10(a) shows the dependence on this angle of the average number of reflections prior to absorption. Note three distinct azimuthal regions. The number of reflections prior to absorption is relatively low on the outer wall $\left(\left|\Phi_{180}\right|<1.5^{\circ}\right)$, since this narrow azimuthal region has a direct line of sight with the (unreflected) synchrotron radiation. The average number of reflections prior to absorption is roughly constant across the top and bottom of the chamber $\left(1.5^{\circ}<\left|\Phi_{180}\right|<165^{\circ}\right)$, and it falls again on the inner wall $\left(\left|\Phi_{180}\right|>165^{\circ}\right)$. Figures $10(\mathrm{~b})-10(\mathrm{~d})$ show the distributions in the number of prior reflections for the azimuthal ranges $\left|\Phi_{180}\right|<1.5^{\circ}$, $1.5^{\circ}<\left|\Phi_{180}\right|<165^{\circ}$, and $\left|\Phi_{180}\right|>165^{\circ}$, respectively. In the region $\left|\Phi_{180}\right|<1.5^{\circ}$, most of the photons (83\%) were not reflected prior to absorption.

Due to the correlation of azimuthal angle with number of reflections, and the dependence of the reflectivity on photon energy, one expects a correlation of photon energy with azimuthal angle. The dependence of absorbed photon energy on azimuth is shown in detail in Fig. 11. Since the probability for electron emission and the energy of the emitted electron depend on photon energy, and the energy of the absorbed photon depends on azimuthal angle, we find that the effective quantum efficiency (that is, the efficiency with which an incident photon emits an electron) depends strongly on azimuthal angle.

Figures 11(b)-11(d) illustrate the reason for choosing three distinct azimuthal regions when providing electron energy distributions to the electron cloud buildup simulation, and show the average energy of the absorbed photons in the three azimuthal ranges $\left|\Phi_{180}\right|<1.5^{\circ}, 1.5^{\circ}<\left|\Phi_{180}\right|<165^{\circ}$ and $\left|\Phi_{180}\right|>165^{\circ}$ is 2987,195 and $343 \mathrm{eV}$, respectively, averaged over the full ring.
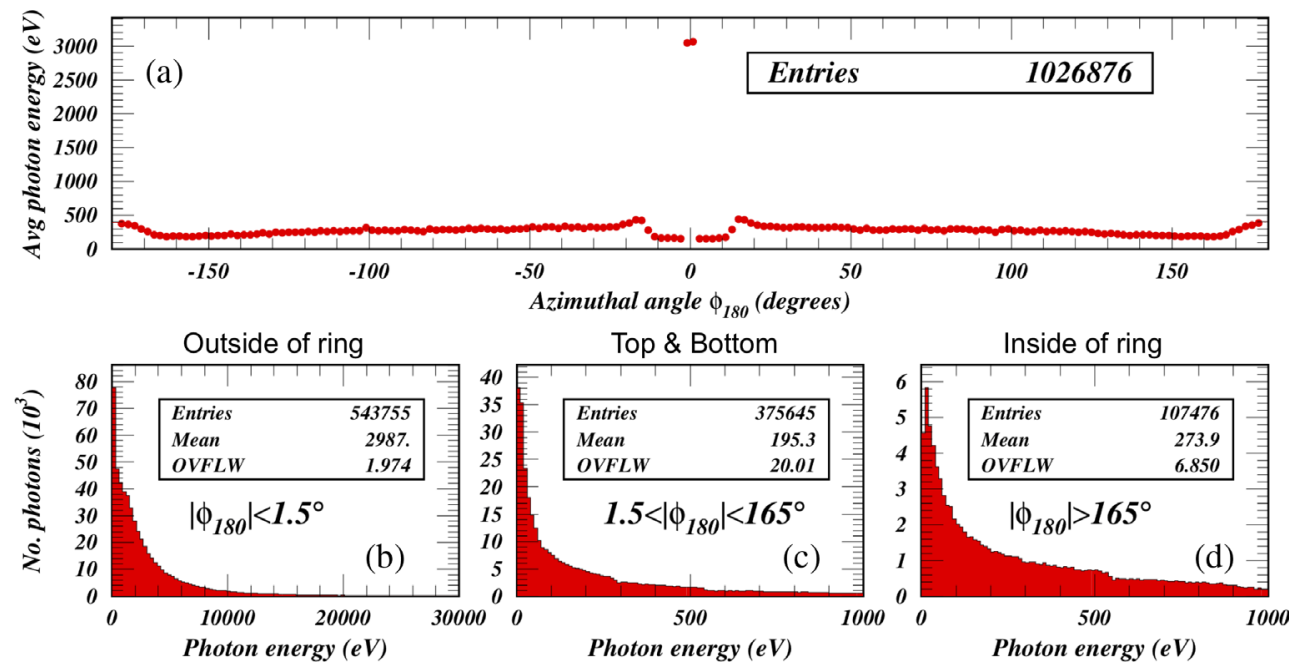

FIG. 11. Average energy of the absorbed photons summed over (a) the full ring as a function of the azimuthal absorption location on the vacuum chamber wall, $\Phi_{180}$. The photon energy distributions are also shown for the three azimuthal regions for which electron energy distributions were provided to the electron cloud buildup simulation: (b) $\left|\Phi_{180}\right|<1.5^{\circ}$, (c) $1.5^{\circ}<\left|\Phi_{180}\right|<165^{\circ}$, and (d) $\left|\Phi_{180}\right|>165^{\circ}$. (Beam energy is $5.3 \mathrm{GeV}$ ). 
We will see below in the section on the GeANT4 simulations that the photoelectron production energy distribution is strongly correlated with the angle of incidence of the photon on the chamber wall. Figures 12 and 13 show details of the photon grazing angle distributions as functions of azimuthal impact location, summed over the fieldfree and dipole regions of the ring, respectively. The distributions in photon angle of incidence on the vacuum chamber wall are somewhat different for the field-free and dipole regions, with important consequences for the average quantum efficiencies. Generally the photons absorbed in the field-free regions have been multiply reflected and are of lower energy, which enhances the quantum efficiency. The details of the vacuum chamber geometry, such as in gate valves, sliding joints and exit windows, result in a complicated pattern of photon incident angles around the ring.

The photon tracking simulation thus provides the longitudinal and transverse absorption location, and incident angle and energy on a photon-by-photon basis. Figure 14 shows the absorbed photon rate in units of photons $/\left(\mathrm{m} \cdot \mathrm{e}^{+} \cdot\right.$ radian $)$ as a function of transverse azimuthal absorption location, averaged separately over the (a) field-free and (b) dipole regions of the ring. The cases of a vacuum chamber material
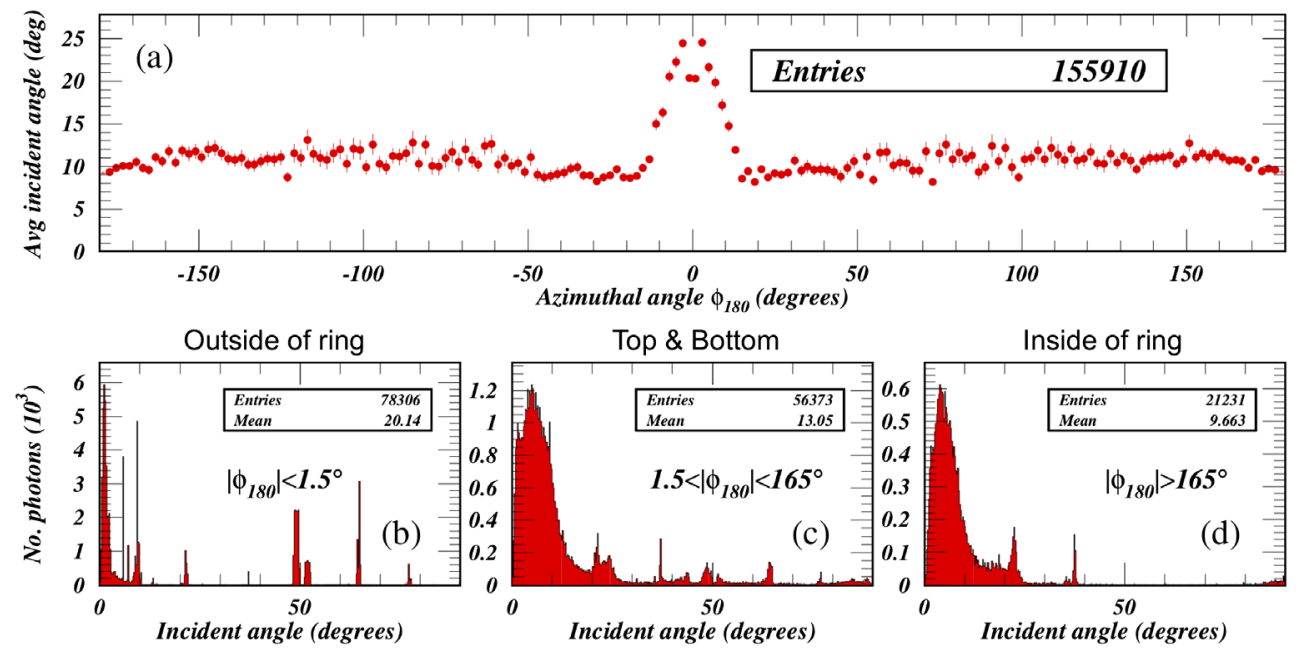

FIG. 12. Average angle of incidence (grazing angle) $\left\langle\theta_{\gamma}^{\text {inc }}\right\rangle$ of the absorbed photons summed over the field-free regions of the CESR ring (a) as a function of the azimuthal location on the vacuum chamber wall, $\Phi_{180}$. The distributions in three azimuthal regions are shown in (b) $\left|\Phi_{180}\right|<1.5^{\circ}$, (c) $1.5^{\circ}<\left|\Phi_{180}\right|<165^{\circ}$, and (d) $\left|\Phi_{180}\right|>165^{\circ}$. (Beam energy is $5.3 \mathrm{GeV}$ ).
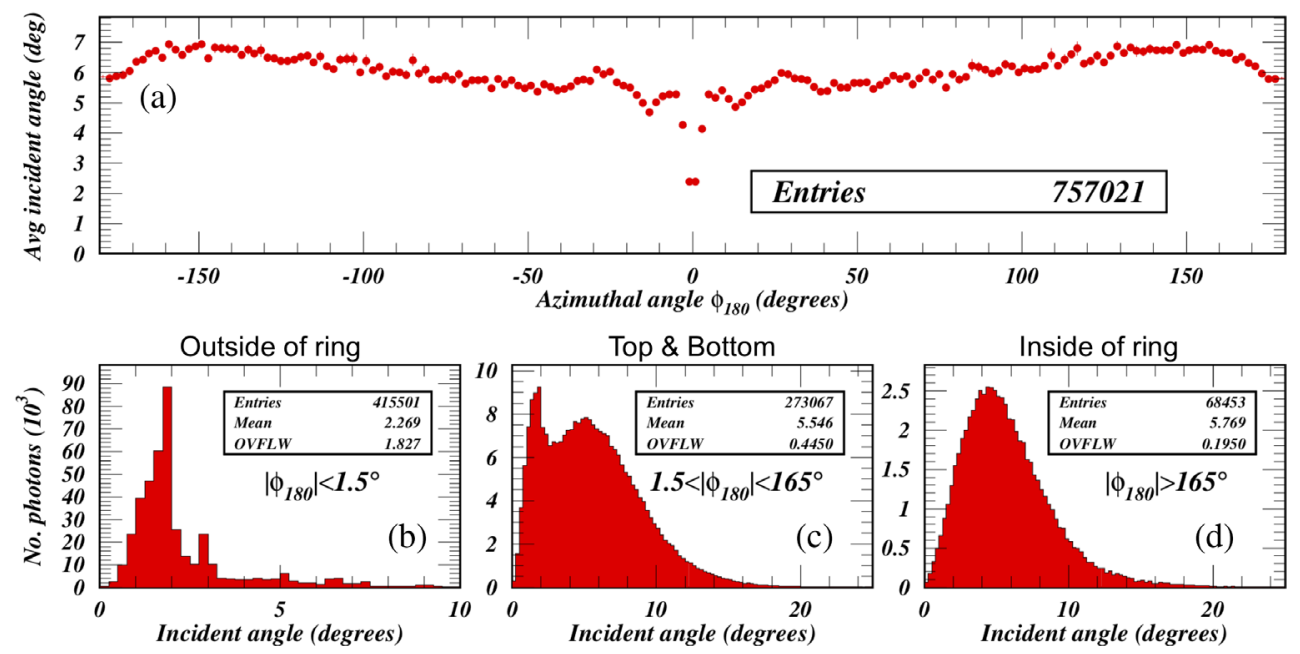

FIG. 13. Average angle of incidence (grazing angle) $\left\langle\theta_{\gamma}^{\text {inc }}\right\rangle$ of the absorbed photons summed over the dipole regions of the CESR ring. These distributions can be compared to those summed over the field-free regions of the ring shown in Fig. 12. The dependence of quantum efficiency on incident photon angle results in significantly different photoelectron production rates in the field-free and dipole regions. (Beam energy is $5.3 \mathrm{GeV}$ ). 


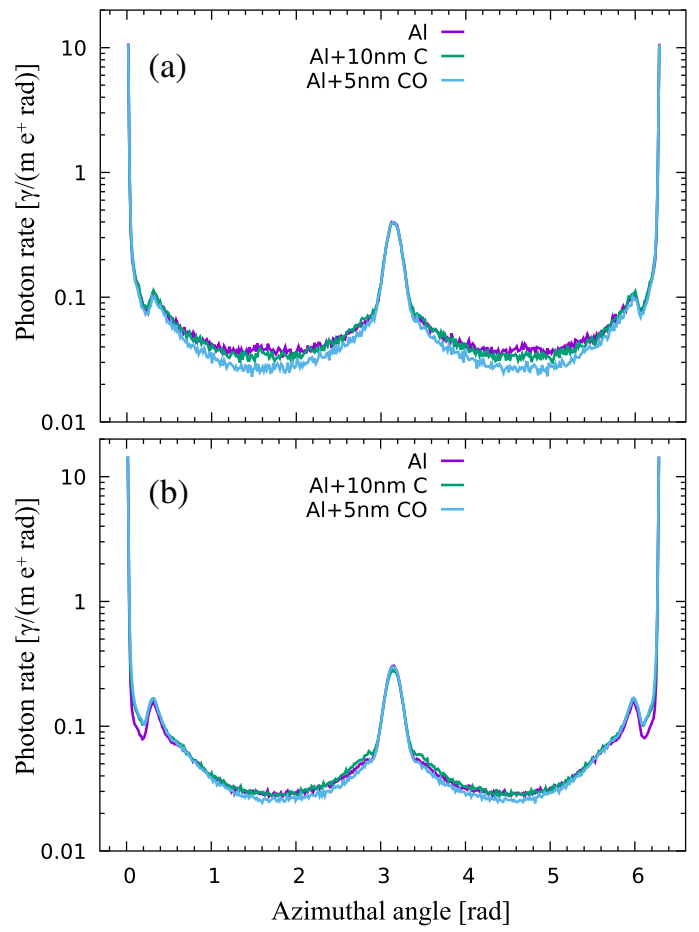

FIG. 14. Azimuthal distribution of photon absorption rate averaged over (a) field-free and (b) dipole regions of the CESR ring. (Beam energy is $5.3 \mathrm{GeV}$.)

consisting of aluminum, aluminum with a 10-nm carbon layer, or aluminum with a 5-nm carbon-monoxide layer are compared.

\section{B. GEANT4-based electron production}

The GEANT4 simulation toolkit $[12,17,18]$ combines theoretical calculations with measurement databases to implement fast tracking and particle interaction algorithms for modeling tasks in experimental particle physics, astrophysics, and medical applications, among others. An extensive bibliography is available [26,27], including articles specifically on low-energy electromagnetic interactions of photons and electrons [28-31] and atomic deexcitation processes $[32,33]$.

\section{Quantum efficiency}

In order to determine the azimuthal dependence of the quantum efficiency, we subdivide the vacuum chamber wall into 720 azimuthal bins. The grazing angle and energy distributions of photons absorbed in each bin are determined by the photon tracking code. Given a sample of photon energies and angles of incidence, the GEANT4 code is used to generate $10^{5}$ photoabsorption events, determining the rate of emitted electrons summed over the bin. Examples of such events are shown in Fig. 15.

We thus obtain a value for the electron production rate specific to the photon incident angle and energy distribution in each azimuthal bin, including (relatively rare)

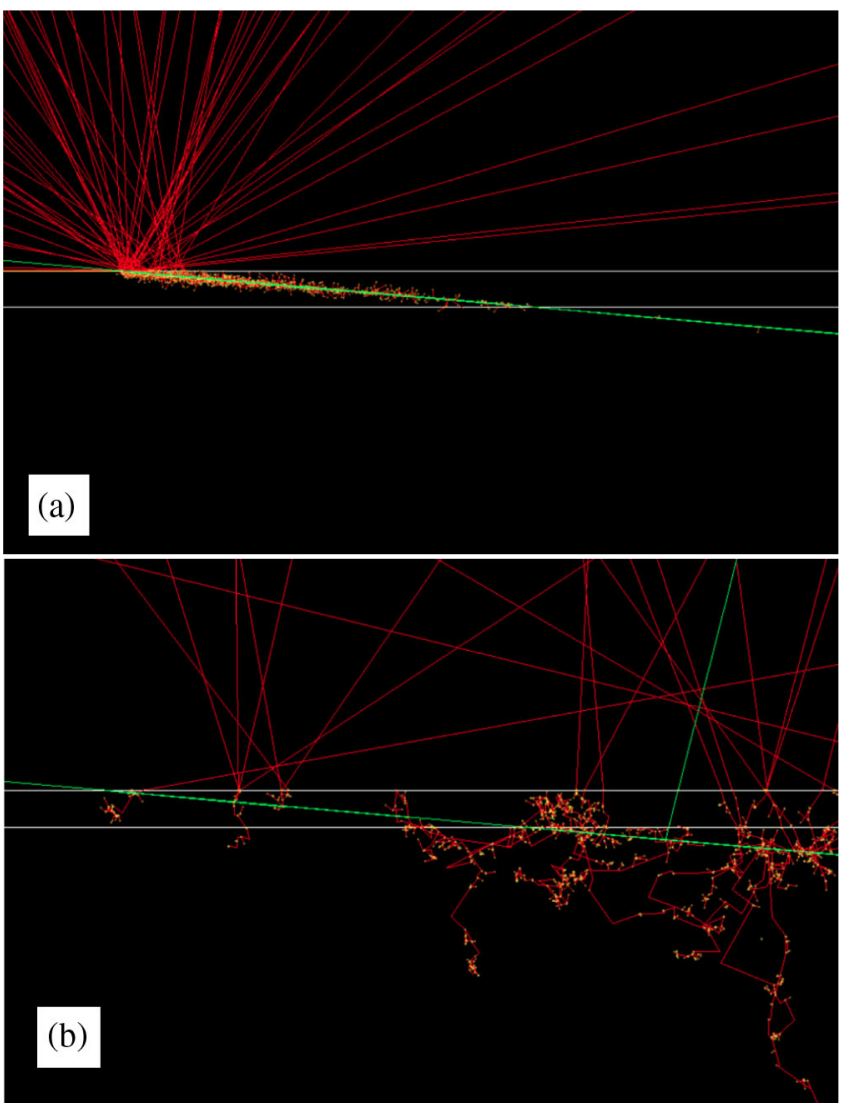

FIG. 15. Tracks from incident photons (green), initially traveling left to right, and subsequently generated electrons (red) in the GEANT4 simulation for photon energies of (a) $30 \mathrm{eV}$ and (b) $2 \mathrm{keV}$. Low-energy photons interact primarily with the 5-nm $\mathrm{CO}$ layer, while the higher energy photons interact in the aluminum. Electrons produced by photoeffect reach the interior of the vacuum chamber via rescattering, while those produced radially symmetrically by atomic deexcitation processes can exit the wall more directly.

multielectron production events. Figure 16 exemplifies the detail with which GEANT4 calculates average electron production rates for various wall materials. Sharp enhancements in electron production are shown for photon energies at the atomic shell transition energies, such as aluminum $\mathrm{L}_{\mathrm{II}}$ and $\mathrm{L}_{\mathrm{III}}(73 \mathrm{eV})$, carbon $\mathrm{K}(284 \mathrm{eV})$, oxygen $\mathrm{K}(543 \mathrm{eV})$, and aluminum $\mathrm{K}(1560 \mathrm{eV})$.

The strong dependence of the quantum efficiency on the incident angle of the absorbed photon in the GEANT4 modeling is illustrated in Fig. 17, favoring more grazing angles. We recall that the average incident angle of the absorbed photons in the azimuthal ranges $\left|\Phi_{180}\right|<1.5^{\circ}$, $1.5^{\circ}<\left|\Phi_{180}\right|<165^{\circ}$ and $\left|\Phi_{180}\right|>165^{\circ}$ are $20.14^{\circ}, 13.05^{\circ}$ and $9.66^{\circ}\left(2.27^{\circ}, 5.55^{\circ}\right.$, and $\left.5.77^{\circ}\right)$, in the field-free (dipole) regions, respectively, for the case of the $5.3 \mathrm{GeV}$ positron beam.

Figure 18 shows azimuthal distributions in average quantum efficiency obtained from the GEANT4 simulations for the $5.3 \mathrm{GeV}$ positron beam. The resulting distributions 


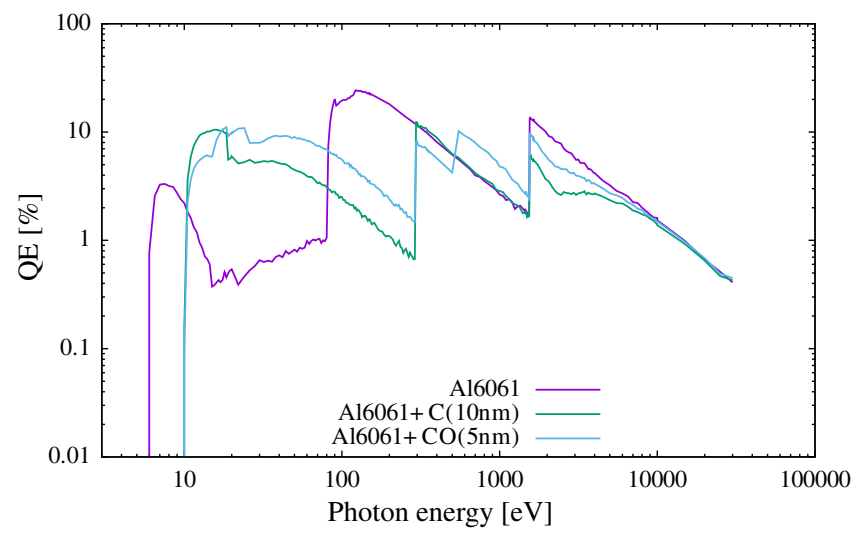

FIG. 16. Quantum efficiency versus photon energy for photons incident at a $5^{\circ}$ grazing angle, for the aluminum alloy 6061 , aluminum with carbon layer, and aluminum with carbon monoxide layer. The quantum efficiency is sharply enhanced at photon energies above various atomic shell transition energies, such as aluminum $\mathrm{L}_{\mathrm{II}}$ and $\mathrm{L}_{\mathrm{III}}(73 \mathrm{eV})$, carbon $\mathrm{K}(284 \mathrm{eV})$, oxygen $\mathrm{K}(543 \mathrm{eV})$, and aluminum $\mathrm{K}(1560 \mathrm{eV})$.

in electron production rate in the 720 azimuthal bins provided to the electron cloud buildup simulation code for the case of the aluminum chamber with the 5-nm CO layer are shown in Fig. 19. The integrated rates are 0.0454 and 0.0839 electrons $/\left(\mathrm{m} \cdot \mathrm{e}^{+}\right)$for the field-free and dipole regions, respectively. Prior to our development work, the photoelectron seeding for the EC buildup simulation code was characterized exclusively in terms of these two integrated rates and two values for effective average reflectivity around the ring [19].

\section{Photoelectron energy distributions}

In addition to the determination of quantum efficiencies, we obtain energy distributions of the photoelectrons in each of the three azimuthal regions defined above, $\left|\Phi_{180}\right|<1.5^{\circ}$,

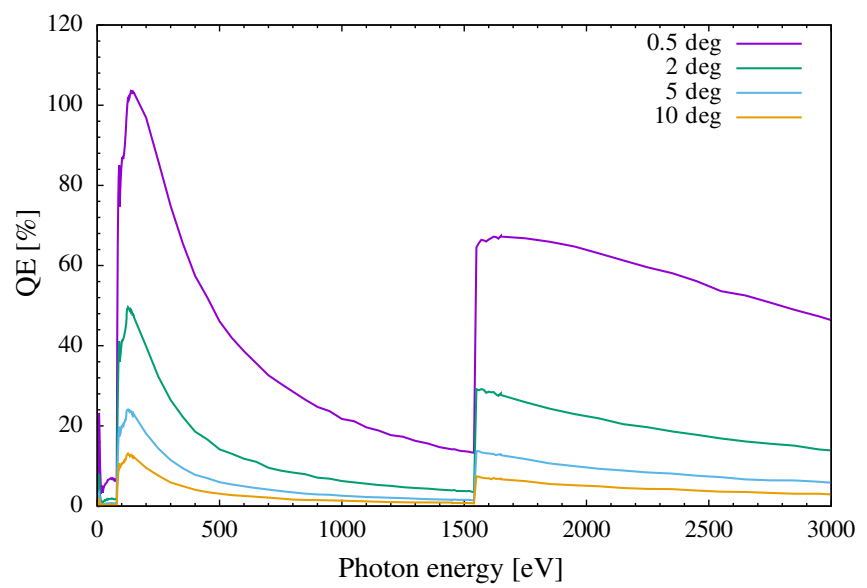

FIG. 17. Quantum efficiency versus photon energy for photons incident at grazing angles between $0.5^{\circ}$ and $10^{\circ}$ for the aluminum alloy 6061 as modeled in GEANT4.
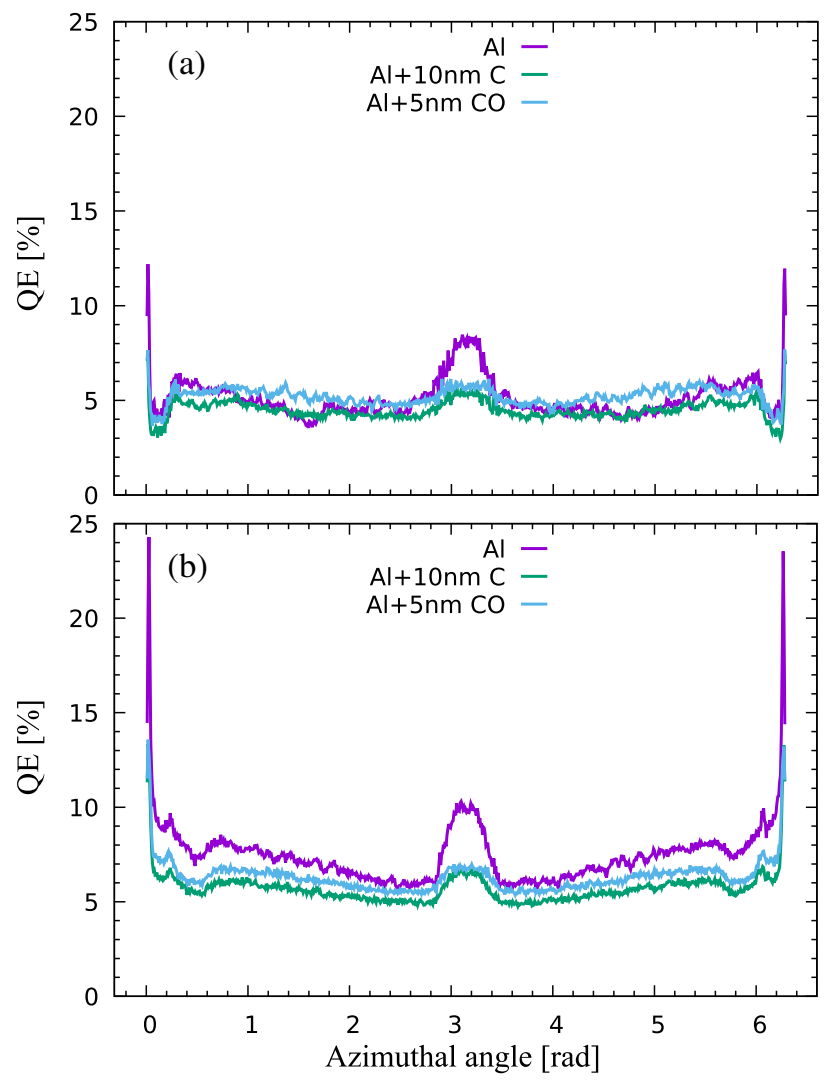

FIG. 18. Azimuthal dependence of quantum efficiency for (a) field-free regions and (b) dipole regions of the CESR ring for aluminum and aluminum with a carbon or carbon monoxide layer. (Beam energy is $5.3 \mathrm{GeV}$.)

$1.5^{\circ}<\left|\Phi_{180}\right|<165^{\circ}$ and $\left|\Phi_{180}\right|>165^{\circ}$ by simulating $10^{6}$ events in each region, again with GEANT4 simulations using absorbed photon data from the photon tracking code. These distributions are shown for the CESR dipole regions in Fig. 20. Within each of these three angular regions,

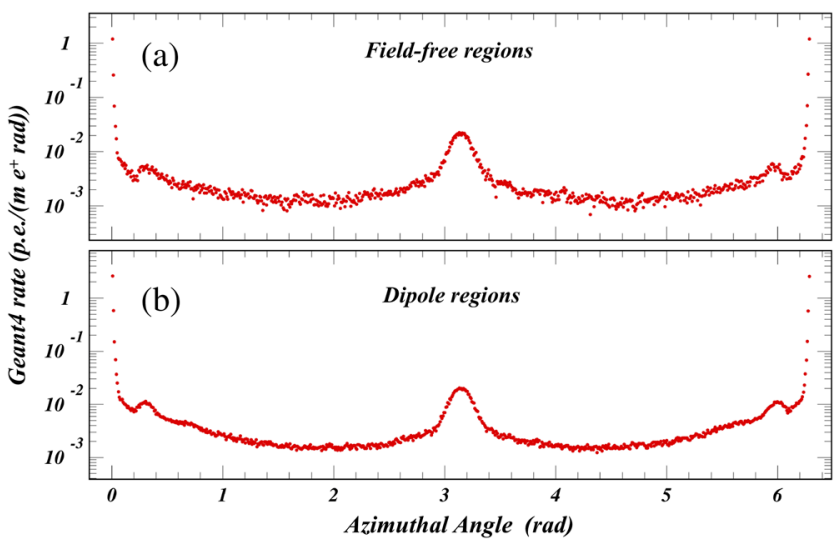

FIG. 19. Electron production rates as a function of azimuthal production location on the vacuum chamber wall for (a) field-free regions and (b) dipole regions in units of electrons $/\left(\mathrm{m} \cdot \mathrm{e}^{+} \cdot\right.$ radian $)$. (Beam energy is $5.3 \mathrm{GeV}$.) 


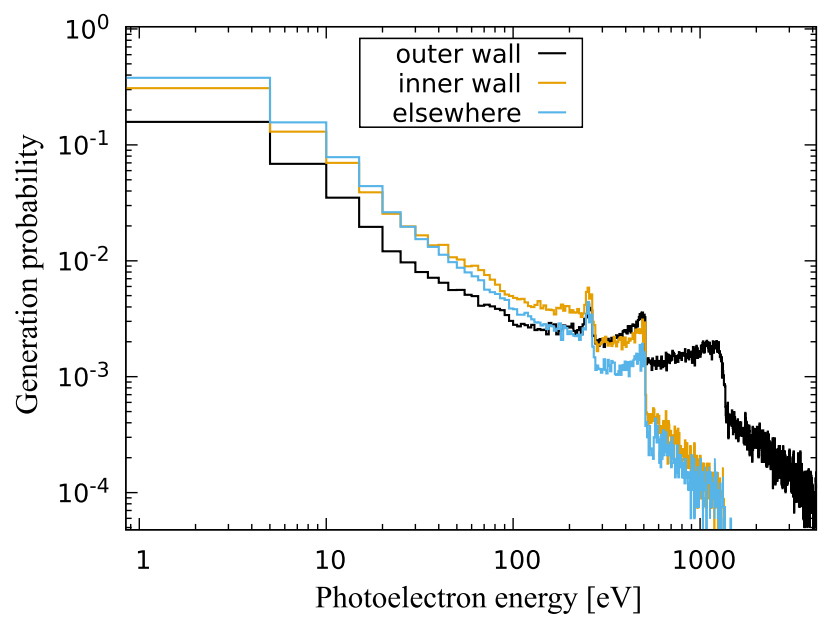

FIG. 20. The energies of photoelectrons emitted from the inner wall and elsewhere are lower than those emitted from the outside wall. The plotted distributions are summed over the dipole regions. Since lower energy photons are more likely to be reflected, and the inner wall and elsewhere (including top and bottom) are struck exclusively by reflected photons, the energy of the photoelectrons is likewise lower. These distributions are used as input to the electron cloud buildup simulations. (Beam energy is $5.3 \mathrm{GeV}$.)

electron energy distribution is roughly independent of azimuthal angle. The quantum efficiency values and photoelectron energy distributions are obtained separately for the field-free and dipole regions of the ring, resulting in a total of $1.5 \times 10^{8}$ simulated events to provide input to the electron cloud buildup simulations.

The simulation results for the photoelectron energy distributions show substantial high-energy tails, resulting in an average energy in the azimuthal ranges $\left|\Phi_{180}\right|<1.5^{\circ}$, $1.5^{\circ}<\left|\Phi_{180}\right|<165^{\circ}$ and $\left|\Phi_{180}\right|>165^{\circ}$ of 761,99 and $120 \mathrm{eV}(662,78$ and $110 \mathrm{eV})$, for the field-free (dipole) regions, respectively. These distributions are sensitive to the atomic level thresholds satisfied by the absorbed photon energy distributions. The principal source of the highenergy electrons are atomic deexcitation processes such as the Auger effect. Both the high-energy electrons and those emitted from the wall at low energies after multiple scattering show similar, semispherically symmetric exit angle distributions, so this was used in the modeled emission angles. These three energy distributions as well as the average electron production rates in $0.5^{\circ}$ azimuthal bins are provided separately for the field-free and dipole regions of the CESR ring as input to the electron cloud buildup calculations. Our modeling has shown that it is important, and to an accuracy acceptable, for modeling the measurement results, sufficient, to differentiate between the field-free and dipole-occupied regions, comprising $17 \%$ and $66 \%$ of the ring, respectively. Buildup simulations in quadrupole and other magnetic field environments show the contribution to the simulated tune shift values from the remaining $17 \%$ of the ring to be at the level of a percent. In quadrupoles, electrons are constrained to migrate in small regions along the field lines to the poles where they are absorbed, leading to a rapid attenuation of the cloud. Simulations indicate that the density of the cloud along the trajectory of the beam is small in quadrupole fields [34]. The large-aperture electrostatic separators and rf cavities are also excluded.

The energy distribution of produced electrons is of particular importance, since the modeled and measured betatron tune shifts show a strong dependence on beam bunch population between $0.64 \times 10^{10}$ and $9.6 \times 10^{10}$ positrons/bunch. The associated beam kicks for electrons produced at the wall can be comparable to the electron production energies. These GEANT4 simulations show that the primary sources of high-energy electrons $(>100 \mathrm{eV})$ are atomic deexcitation processes, such as the Auger effect. The contribution of such electrons to cloud development is greater at lower bunch population, since their kinetic energies provide for higher subsequent SEY, replacing the effect of strong momentum kicks from the beam bunches. Figure 21 shows a schematic diagram of the CESR vacuum chamber illustrating the beam kick quantities in Table II. In an impulse approximation, the beam bunch charge integrated over the bunch passage gives the momentum kick to an electron produced at the wall [35]. An electron generated simultaneously with the passage of the longitudinal center of the bunch, e.g., receives half of this kick. In Table II, we present the kick as the kinetic energy gained by the electron during the bunch passage. The elliptical shape of the vacuum chamber results in an increased (reduced) kick in the vertical (horizontal) plane

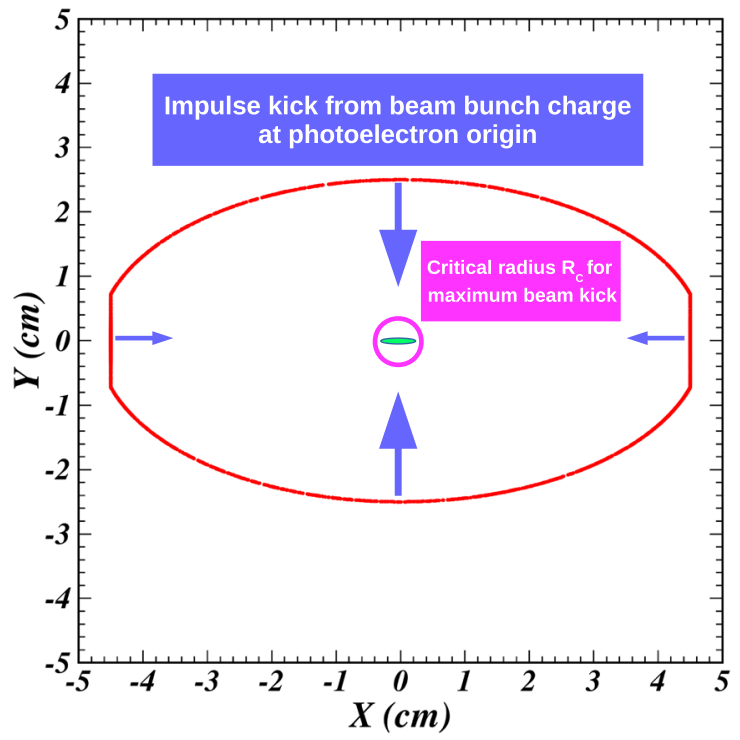

FIG. 21. Schematic diagram of the laterally truncated elliptical CESR vacuum chamber illustrating the beam kicks for an electron produced at the wall and the radius $R_{\mathrm{C}}$ at which an electron receives the maximum kick. Examples of these quantities are given in Table II. 
TABLE II. Parameters for the acceleration provided by a positron bunch to a cloud electron located at the vacuum chamber wall on the $X$ or $Y$ axes. These examples correspond to the CESRTA measurements of betatron tune shifts as well as for the predictions for the $6.0 \mathrm{GeV}$ upgrade of CESR [8,36]. Kick values for the case of the field-free regions of the ring are shown. The total kick values are given as the kinetic energy of the electron following acceleration by the positron bunch in the impulse approximation. The direct and image kick values are signed according to whether they add or subtract from the total kick. The beam sizes shown are averages over the fieldfree, dipole, combined-function (DQ) magnet and compact undulator (CCU) regions of the ring.

\begin{tabular}{|c|c|c|c|c|c|c|c|c|}
\hline \multicolumn{2}{|l|}{ Beam energy $(\mathrm{GeV})$} & \multicolumn{2}{|c|}{2.085} & \multicolumn{3}{|c|}{5.289} & \multicolumn{2}{|c|}{6.000} \\
\hline Beam size $\sigma_{\mathrm{X}} \times \sigma_{\mathrm{Y}} \times \sigma_{\mathrm{Z}}$ & $\begin{array}{l}\text { Drift }(\mathrm{mm}) \\
\text { Dipole }(\mathrm{mm}) \\
\text { DQ magnets }(\mathrm{mm}) \\
\text { CCU undulators }(\mathrm{mm})\end{array}$ & $\begin{array}{l}0.856 \\
0.732\end{array}$ & $\begin{array}{l}27 \times 9.2 \\
26 \times 9.2 \\
\end{array}$ & $\begin{array}{l}1.50 \\
1.44\end{array}$ & $\begin{array}{l}\times 0.142 \times \\
\times 0.139 \times \\
\text { N/A } \\
\text { N/A }\end{array}$ & $\begin{array}{l}15.8 \\
15.8\end{array}$ & $\begin{array}{l}0.875 \times \\
0.889 \times \\
0.219 \times \\
0.566 \times\end{array}$ & $\begin{array}{l}3 \times 15.6 \\
3 \times 15.6 \\
0 \times 15.6 \\
8 \times 15.6\end{array}$ \\
\hline $\begin{array}{l}\text { Bunch population }\left(10^{10}\right) \\
\text { Bunch current }(\mathrm{mA} / \mathrm{bunch}) \\
\text { Critical radius } R_{\mathrm{C}}(\mathrm{mm}) \\
\text { Maximum kick }(\mathrm{keV})\end{array}$ & & $\begin{array}{l}0.64 \\
0.4 \\
0.73 \\
1.2\end{array}$ & $\begin{array}{l}1.12 \\
0.7 \\
0.96 \\
2.5\end{array}$ & $\begin{array}{l}3.25 \\
2.0 \\
2.14 \\
3.5\end{array}$ & $\begin{array}{l}6.66 \\
4.2 \\
3.1 \\
9.0\end{array}$ & $\begin{array}{r}9.5 \\
6.0 \\
3.7 \\
14.1\end{array}$ & $\begin{array}{l}3.52 \\
2.2 \\
2.2 \\
6.1\end{array}$ & $\begin{array}{r}7.11 \\
4.4 \\
3.2 \\
14.0\end{array}$ \\
\hline$X=4.5, Y=0 \mathrm{~cm}$ & $\begin{array}{l}\text { Direct kick }(\mathrm{eV}) \\
\text { Image kick }(\mathrm{eV}) \\
\text { Total kick }(\mathrm{eV})\end{array}$ & $\begin{array}{r}0.16 \\
-0.14 \\
0.02\end{array}$ & $\begin{array}{r}0.5 \\
-0.44 \\
0.06\end{array}$ & $\begin{array}{r}41.8 \\
-41.3 \\
0.5\end{array}$ & $\begin{array}{r}17.6 \\
-15.6 \\
2.0\end{array}$ & $\begin{array}{r}36 \\
-32 \\
4\end{array}$ & $\begin{array}{r}4.9 \\
-4.3 \\
0.6\end{array}$ & $\begin{array}{r}20.0 \\
-17.7 \\
2.3\end{array}$ \\
\hline$X=0, Y=2.5 \mathrm{~cm}$ & $\begin{array}{l}\text { Direct kick }(\mathrm{eV}) \\
\text { Image kick }(\mathrm{eV}) \\
\text { Total kick }(\mathrm{eV})\end{array}$ & $\begin{array}{l}0.50 \\
0.60 \\
1.10\end{array}$ & $\begin{array}{l}1.6 \\
1.6 \\
3.2\end{array}$ & $\begin{array}{l}13.4 \\
13.9 \\
27.3\end{array}$ & $\begin{array}{r}56 \\
59 \\
115\end{array}$ & $\begin{array}{l}115 \\
120 \\
235\end{array}$ & $\begin{array}{l}15.8 \\
16.3 \\
32.1\end{array}$ & $\begin{array}{r}64.4 \\
66.5 \\
130.9\end{array}$ \\
\hline
\end{tabular}

from the image charges which ensure the boundary conditions at the wall. The transverse beam size determines the critical radius $R_{\mathrm{C}}$ at which a cloud electron receives the maximum kick during bunch passage. Table II shows these values for the bunch populations and beam sizes for which CESRTA betatron tune shift measurements are available, and also for the parameters of the upgraded Cornell High Energy Synchrotron Source to be commissioned at $6 \mathrm{GeV}$ in 2019 [14]. The kick corresponding to wall-to-wall traversal of cloud electrons between bunch passages depends on the bunch separation. For 14-ns bunch spacing the kick for horizontal (vertical) wall-to-wall traversal prior to arrival of the succeeding bunch is $36 \mathrm{eV}(9 \mathrm{eV})$. Another relevant consideration in this regard is that SEY is maximum for an electron carrying an energy of about $300 \mathrm{eV}$.

The wide range of beam kick values causes a great variation in the cloud dynamics as a function of bunch population as evidenced by the patterns of observed tune shifts. The interplay between these kicks and the electron production energy distribution is an important aspect of the cloud buildup, especially when they result in cloud electron energies on the steeply rising slope of the SEY curve. The effects of the photoelectron production energy distribution are particularly pronounced at low bunch populations such as those for the $2.1 \mathrm{GeV}$ data, where we have observed changes in the modeled tune shifts of about $30 \%$ when only low-energy $(\simeq 5 \mathrm{eV})$ photoelectrons are included in the simulations.

\section{Electron cloud buildup}

The EC buildup simulation is based on an extended version [5] of the ECLOUD [37] code. The number of primary electrons created by each beam particle, along with energy and angular distribution, is input to the buildup simulation. That information is derived from the photon tracking and electron production simulations described in the previous sections. The buildup of the cloud is largely determined by the emission of secondary electrons from the walls of the vacuum chamber. In these simulations, the phenomenology of the SEY physics is a parametrized Furman-Pivi model [22]. The SEY parameters are fit to data as described in Sec. III D. Additional inputs to the buildup simulation include beam size and bunch population as given in Table II. The beam sizes used in these simulations for the $2.085,5.289$, and $6.0 \mathrm{GeV}$ beams are averaged over the field-free, dipole, combined-function (DQ) and compact undulator $(\mathrm{CCU})$ regions of the ring. The large ring-averaged horizontal size is dominated by dispersion. In these simulations we clearly see the pinch effect of the beam attracting the EC (Fig. 22). We find that cloud buildup is rather insensitive to the beam size, and that using ring-averaged values per element type is a sufficient approximation. The following figures in this section characterizing the cloud buildup model use the example of the $2.1 \mathrm{GeV}$ simulations.

Electric field maps on a $15 \times 15$ grid of $\pm 5 \sigma$ of the transverse beam size are obtained for 11 time slices as the bunch passes through the cloud. The time interval between slices is 20 ps. Figure 23 shows these field maps in a dipole for bunch number 30 in the $0.7 \mathrm{~mA} /$ bunch train during the central time slice. Since only a small fraction $(\sim 0.1 \%)$ of photoelectrons are within the $\pm 5 \sigma$ region around the beam, it is necessary to combine the results of many ECLOUD simulations to achieve sufficient statistical accuracy in the calculation of the electric field. 

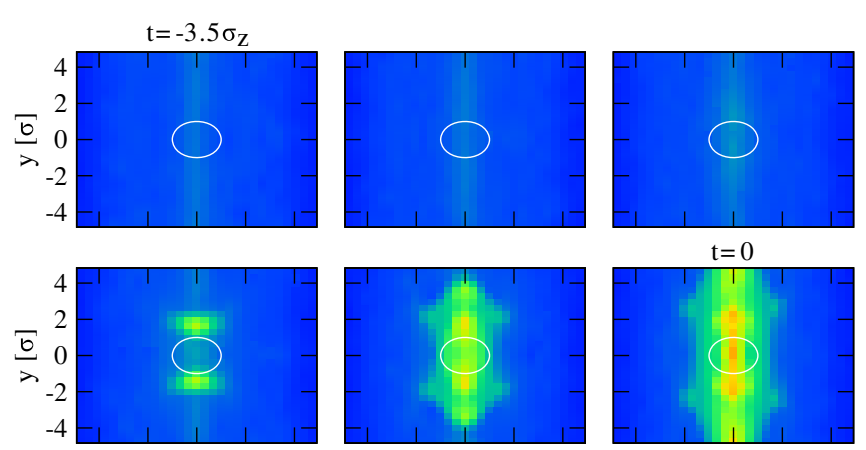

$\mathrm{t}=0$
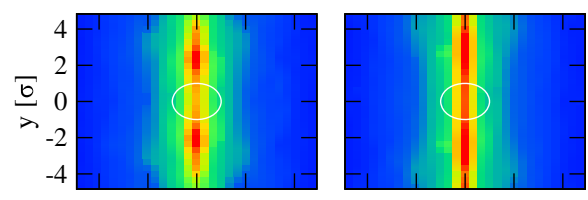

$\mathrm{t}=+3.5 \sigma_{\mathrm{Z}}$
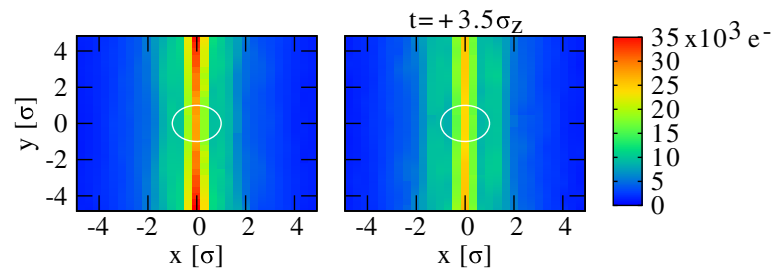

FIG. 22. Transverse charge distributions of the electron cloud in an 800-Gauss dipole field during the passage of the last bunch of the 30-bunch train at $0.7 \mathrm{~mA} /$ bunch at $2.1 \mathrm{GeV}$, in the central region ( $\pm 5 \sigma$ of the beam size) for 11 time slices spanning $\pm 3.5 \sigma_{z}$. The rms beam size is shown as a white circle. Time increases from left to right, top to bottom. The time between slices is $20 \mathrm{ps}$. (Beam energy is $2.1 \mathrm{GeV}$.)

The modeled horizontal tune shift values are calculated from the cloud space-charge electric field gradients according to

$$
\Delta Q_{x}=f_{\text {rev }} \frac{e}{4 \pi E_{\text {beam }}} \oint \beta_{x}\left\langle\frac{\mathrm{d} E_{\mathrm{X}}}{\mathrm{d} x}\right\rangle_{\text {beam }} \mathrm{d} s
$$

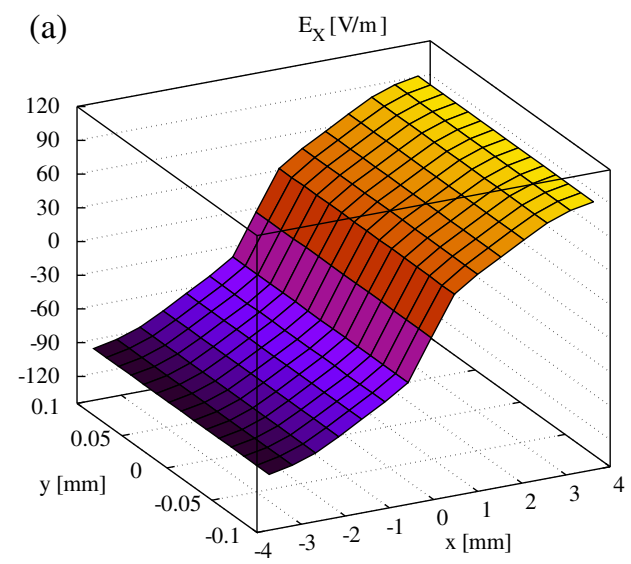

where $f_{\text {rev }}$ is the revolution frequency of $390 \mathrm{kHz}, e$ is the electron charge, $E_{\text {beam }}$ is the beam energy, and $\left\langle\mathrm{d} E_{\mathrm{X}} / \mathrm{d} x\right\rangle_{\text {beam }}$ is the electric field gradient averaged over the transverse charge distribution of the beam. The vertical tune shifts are calculated similarly. The positive signs of the measured horizontal and vertical tune shifts (Figs. 2 and 3) indicate that $\vec{\nabla} \cdot \vec{E} \neq 0$ and that it is cloud electrons in the path of the positron bunch that are largely responsible for the tune shift. The like-sign behavior is similar to the beambeam tune shift in an electron-positron collider.

The integral over ring circumference is approximated as a sum over the field gradient calculated for each element type weighted by its ring occupancy fraction. The beta function factor is approximated as an average of the beta function over each element type in the ring. Table III shows modeling results and parameter values for each of the tune shift calculations. For the upgraded light source operation at $6 \mathrm{GeV}$, contributions from the newly introduced DQ magnets and the CCU undulators were included. The CCU magnetic field was modeled as a dipole field.

The pinch effect, whereby the bunch attracts the nearby cloud as it passes, can clearly be seen in Figs. 24 and 25 as a dramatic increase in the modeled electric field gradients during the bunch passage. Figure 26 shows the measured tune shift in each of the 30 bunches in the train as well as for witness bunches positioned one at a time with some delay beyond the end of the train. Unlike the measured tune shifts along the train, which are referenced to that of the first bunch in the train and where the bunch populations are equal at a level of better than $1 \%$, the observed witness bunch tune shifts require a correction for the ring impedance contribution to the coherent tune shift, which has been measured to be about $-1 \mathrm{kHz} / \mathrm{mA}$ [38]. The cloud-induced tune shifts of the witness bunches are observed to be independent of witness bunch current, whereas the pinch by definition is not. These measurements clearly show that the pinch effect does not contribute to the tune shift. For this

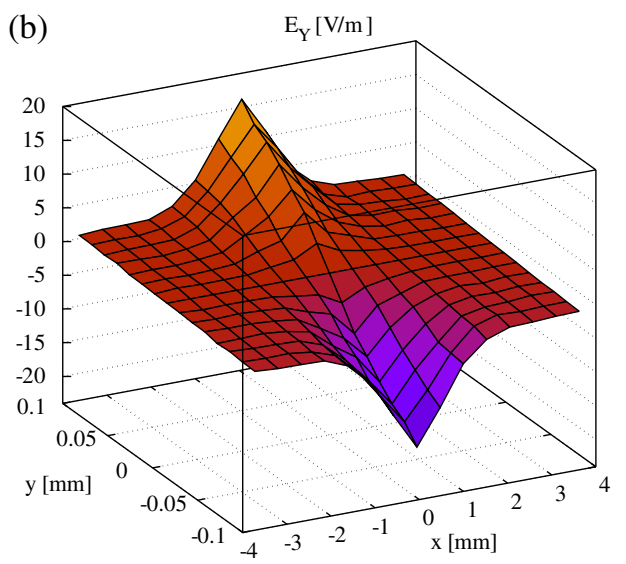

FIG. 23. Space-charge electric field maps (a) $E_{\mathrm{X}}(x, y)$ and (b) $E_{\mathrm{Y}}(x, y)$ in a region of $\pm 5 \sigma$ of the transverse beam size for the central time slice of the last bunch of the 30 bunch train at $0.7 \mathrm{~mA} /$ bunch at $2.1 \mathrm{GeV}$, corresponding to the sixth picture in Fig. 22. (Beam energy is $2.1 \mathrm{GeV}$.) 
TABLE III. Modeling results and parameter values used in each of the simulated tune shift calculations. The vacuum chamber shapes used in the EC buildup simulations are approximately elliptical with vertical side walls, except for the undulator chambers, which are rectangular. The numbers of photons and electrons refer to the total numbers generated in the simulation.

\begin{tabular}{|c|c|c|c|c|c|c|c|c|}
\hline \multirow[t]{2}{*}{ Beam energy $(\mathrm{GeV})$} & \multicolumn{2}{|c|}{2.085} & \multicolumn{2}{|c|}{5.289} & \multicolumn{4}{|c|}{6.000} \\
\hline & Field-free & Dipole & Field-free & Dipole & Field-free & Dipole & DQ magnet & $\mathrm{CCU}$ \\
\hline Ring fraction $(\%)$ & 16.3 & 65.7 & 16.3 & 65.7 & 57.1 & 23.1 & 3.7 & 2.9 \\
\hline Number of photons & $1.72 \times 10^{5}$ & $7.10 \times 10^{5}$ & $1.56 \times 10^{5}$ & $7.57 \times 10^{5}$ & $7.64 \times 10^{5}$ & $3.26 \times 10^{6}$ & $3.37 \times 10^{5}$ & $7.82 \times 10^{4}$ \\
\hline Photon absorption rate $\left[\gamma /\left(\mathrm{m} \cdot \mathrm{e}^{+}\right)\right]$ & 0.378 & 0.370 & 0.728 & 0.876 & 0.833 & 0.973 & 1.655 & 0.3076 \\
\hline Number of electrons & $3.65 \times 10^{6}$ & $4.54 \times 10^{6}$ & $3.74 \times 10^{6}$ & $4.55 \times 10^{6}$ & $3.88 \times 10^{6}$ & $4.58 \times 10^{6}$ & $4.65 \times 10^{6}$ & $4.49 \times 10^{6}$ \\
\hline Electron production rate $\left[\right.$ p.e. $\left./\left(\mathrm{m} \cdot \mathrm{e}^{+}\right)\right]$ & 0.02137 & 0.03144 & 0.0454 & 0.0839 & 0.0603 & 0.0956 & 0.1241 & 0.0317 \\
\hline$\left\langle\beta_{\mathrm{x}}\right\rangle(\mathrm{m})$ & 16.80 & 16.50 & 18.00 & 17.00 & 14.10 & 13.10 & 1.77 & 11.07 \\
\hline$\left\langle\beta_{\mathrm{y}}\right\rangle(\mathrm{m})$ & 24.40 & 22.90 & 21.85 & 21.70 & 18.10 & 19.60 & 15.70 & 3.49 \\
\hline \multicolumn{9}{|l|}{ EC buildup model input parameters: } \\
\hline $\begin{array}{l}\text { Vacuum chamber size }(\mathrm{H} \times \mathrm{V})(\mathrm{mm}) \\
\text { Dipole field }(\mathrm{T})\end{array}$ & \multicolumn{2}{|c|}{$90 \times 50$} & \multicolumn{2}{|c|}{$90 \times 50$} & $\begin{array}{c}90 \times 50 \\
0\end{array}$ & $\begin{array}{l}90 \times 50 \\
0.2277\end{array}$ & $\begin{array}{l}50 \times 22 \\
0.6509\end{array}$ & $\begin{array}{c}50 \times 4.5 \\
1.0000\end{array}$ \\
\hline Quadrupole field gradient (T/m) & 0 & 0 & 0 & 0 & 0 & 0 & 8.762 & 0 \\
\hline
\end{tabular}

reason, the space-charge electric field gradients immediately prior to the bunch arrival are used when calculating the modeled tune shifts.

Simulations for a bunch offset relative to the train are consistent with this measurement result. For an offset

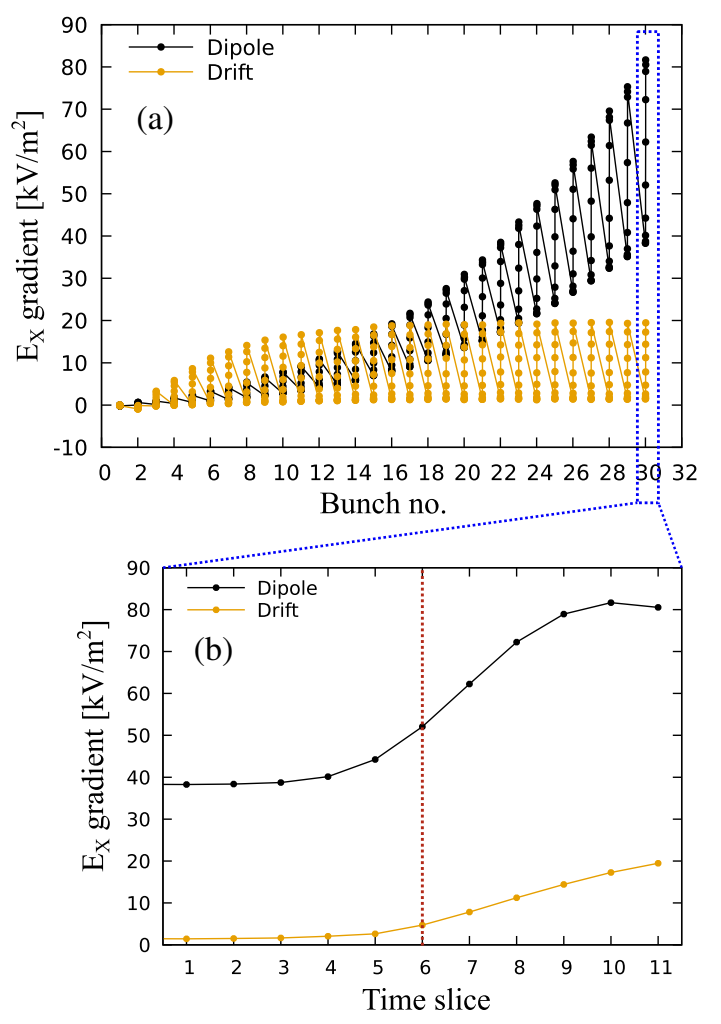

FIG. 24. (a) Horizontal electron cloud space-charge electric field gradients for the 11 time slices within each of 30 bunches for dipoles and field-free regions. (b) Electric field gradients for the 11 time slices in bunch 30, showing the center of the bunch at time slice 6. (Beam energy is $2.1 \mathrm{GeV}$.) bunch (the one being excited) in an on-axis train, the pinched cloud is centered on the offset bunch, even in the presence of a dipole field, as shown in Fig. 27. In the absence of any such beam/cloud offset, the pinch does not contribute any coherent kick to the bunch.

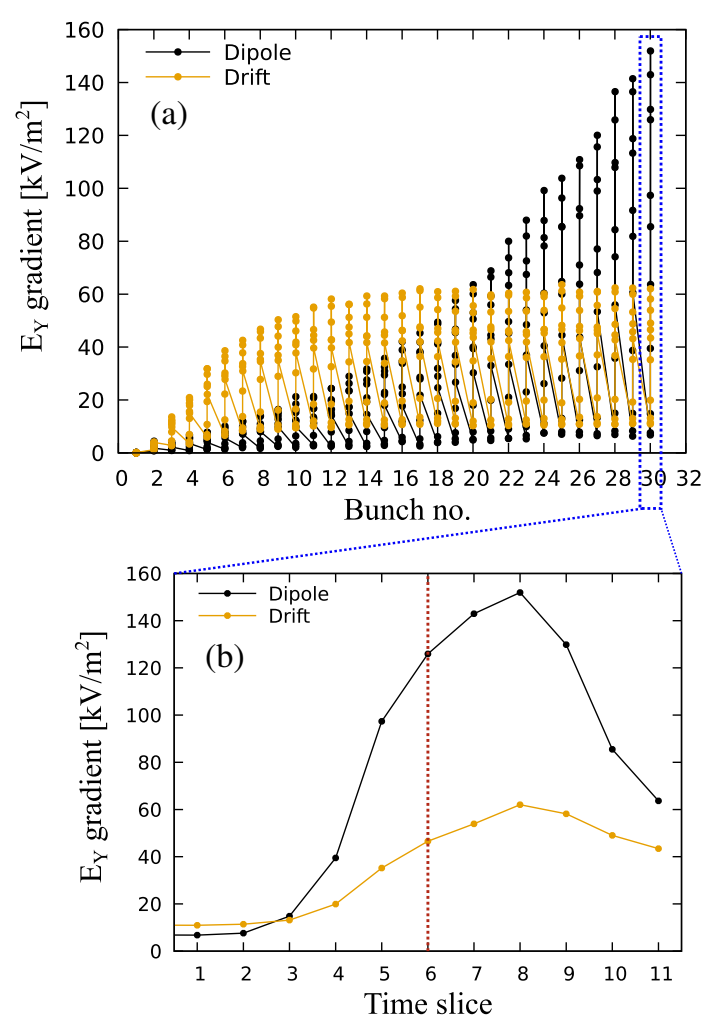

FIG. 25. (a) Vertical electron cloud space-charge electric field gradients for the 11 time slices within each of 30 bunches for dipoles and field-free regions. (b) Electric field gradients for the 11 time slices in bunch 30, showing the center of the bunch at time slice 6. (Beam energy is $2.1 \mathrm{GeV}$.) 


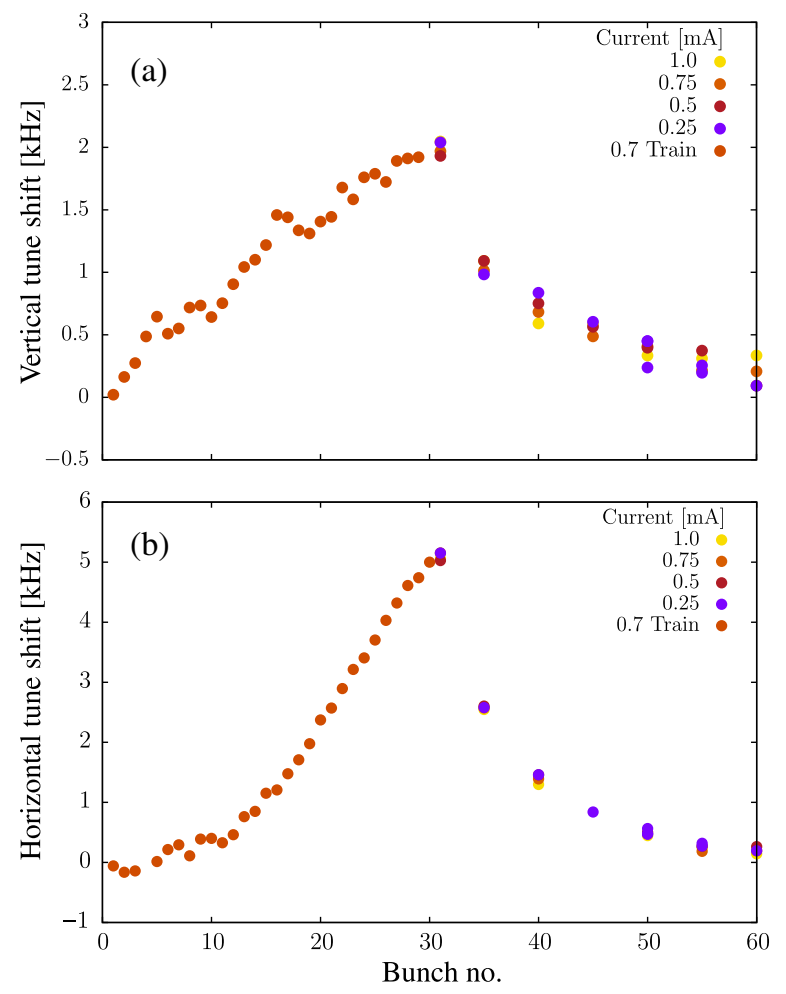

FIG. 26. Tune shifts measured in the (a) vertical and (b) horizontal planes using the tune tracker for a 30-bunch train of positrons at $0.7 \mathrm{~mA} /$ bunch $\left(1.12 \times 10^{10}\right.$ bunch population $)$ at $2.1 \mathrm{GeV}$, followed by a witness bunch in bunch positions 31-60 at currents of $0.25,0.5,0.75$, and $1.0 \mathrm{~mA}$. The vertical tune shift from impedance $(\simeq-1.0 \mathrm{kHz} / \mathrm{mA})$ has been subtracted to show only the contribution from the electron cloud. No dependence of the tune of the witness bunch on the witness bunch current is seen, showing that the pinch effect does not contribute to the tune shift.

\section{SEY parameter determination}

Secondary-electron yield depends on a number of factors, such as incident electron energy and angle, and chamber wall material and coatings (see list below). Tune shifts from simulation are found to depend strongly on these details of the SEY. The effects of the SEY parameters on the tune shifts can be highly correlated. Direct SEY measurements can provide a good starting point, but a comprehensive experimental determination of all the SEY parameters has yet to be obtained. Furthermore, the ring-wide averaged SEY may be different than an external measurement of a vacuum chamber sample. To improve agreement between the model and the tune shift measurements, we use an optimizer to fit the model tune shift values to the measurements, varying a selection of 11 SEY parameters. Measurements of the peak SEY for aluminum show a rapid beam-processing-induced reduction of the peak SEY to a value near $1.8[39,40]$, comparable to the values measured for copper [41]. We therefore use the SEY parameters determined for copper in Ref. [22] as a starting point, rather than the other example given, stainless steel, for which the SEY components are
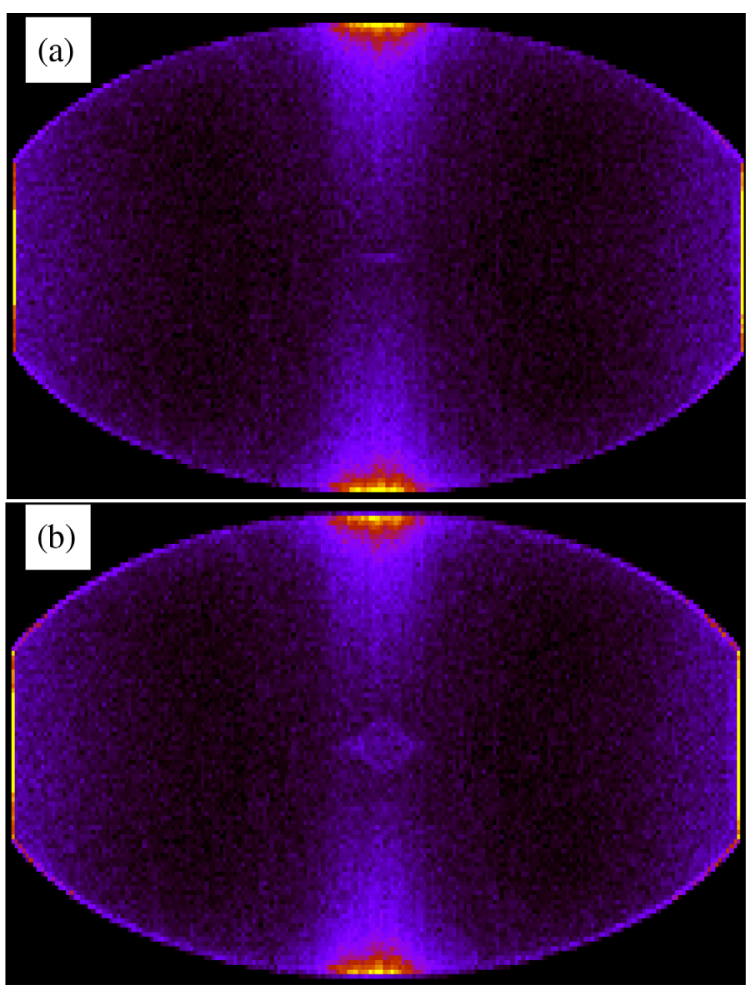

FIG. 27. Simulated electron cloud density during the (a) third and (b) sixth of 11 time slices during of the passage of bunch 15, which has been offset from the centered bunch train by $1 \mathrm{~mm}$ horizontally to simulate the effect of kicking a single bunch when measuring its tune. The "pinched" cloud is found to be centered on the offset bunch position. The short bunch length $(16 \mathrm{~mm})$ bunch results in little effect on the larger built-up cloud. The simulated bunch current is $2 \mathrm{~mA} /$ bunch. At higher currents, the vertical band widens $(4 \mathrm{~mA} /$ bunch $)$ and splits into two (6 mA/bunch). (Beam energy is $5.3 \mathrm{GeV}$.)

rather dissimilar. At each iteration, the EC buildup simulations are run in parallel with the current best SEY parameters, and each parameter increased and decreased by an adaptive increment. The tune shifts from these simulations are obtained, and the Jacobian is calculated and provided to the optimizer. The optimized input parameters are, in the notation of Ref. [22],

(i) $\hat{E}_{\mathrm{ts}}$ : incident electron energy at which the truesecondary yield is maximum for perpendicular incidence,

(ii) $s$ : true-SEY energy dependence parameter, with $\delta_{\mathrm{ts}}\left(\theta_{\mathrm{e}}, E_{0}\right)=\delta_{\mathrm{ts}}\left(\theta_{\mathrm{e}}\right) s x /\left(s-1+x^{s}\right)$, where $x=E_{0} / E_{\mathrm{ts}}\left(\theta_{\mathrm{e}}\right), E_{0}$ being the incident electron energy,

(iii) $P_{1, \mathrm{r}}(\infty)$ : rediffused $\mathrm{SEY}$ at high incident electron energy,

(iv) $\hat{\delta}_{\mathrm{ts}}$ : true-SEY at perpendicular incidence,

(v) $t_{1}$ and $t_{2}:$ amplitude of the cosine dependence and power of the cosine in the true SEY: $\delta_{\mathrm{ts}}\left(\theta_{\mathrm{e}}\right)=\hat{\delta}_{\mathrm{ts}}\left[1+t_{1}\left(1-\cos ^{t_{2}} \theta_{\mathrm{e}}\right)\right]$, where $\theta_{\mathrm{e}}=0^{\circ}$ for perpendicular electron incidence, 
TABLE IV. Initial and optimized SEY parameters, including the sensitivity to each parameter given in the form of an uncertainty calculated from the Jacobian.

\begin{tabular}{lccc}
\hline \hline & Initial & Optimized & Uncertainty \\
\hline$\hat{E}_{\mathrm{ts}}(\mathrm{eV})$ & 277 & 260 & 10 \\
$s$ & 1.54 & 1.58 & 0.05 \\
$P_{1, \mathrm{r}}(\infty)$ & 0.2 & 0.39 & 0.05 \\
$\hat{\delta}_{\mathrm{ts}}$ & 1.88 & 1.53 & 0.04 \\
$t_{1}$ & 0.66 & 0.99 & 0.2 \\
$t_{2}$ & 0.8 & 1.5 & 0.4 \\
$t_{3}$ & 0.70 & 0.77 & 0.50 \\
$t_{4}$ & 1.0 & 1.2 & 1.0 \\
$\epsilon(\mathrm{eV})$ & 1.8 & 3.6 & 0.4 \\
$p$ & 1.0 & 0.8 & 0.2 \\
$\hat{P}_{1, \mathrm{e}}$ & 0.5 & 0.07 & 0.02 \\
\hline \hline
\end{tabular}

(vi) $t_{3}$ and $t_{4}$ : amplitude of the cosine dependence and power of the cosine in true SEY peak energy: $E_{\mathrm{ts}}\left(\theta_{\mathrm{e}}\right)=\hat{E}_{\mathrm{ts}}\left[1+t_{3}\left(1-\cos ^{t_{4}} \theta_{\mathrm{e}}\right)\right]$,

(vii) $\hat{P}_{1, \mathrm{e}}$ : elastic yield in the low-energy limit, and

(viii) $\epsilon$ and $p$ : parameters for the energy distribution of the secondary electrons:

$$
\frac{\mathrm{d} N}{\mathrm{~d} E_{\mathrm{sec}}}\left(E_{\mathrm{sec}}\right) \propto \begin{cases}\frac{\left(E_{\mathrm{sec}} / \epsilon\right)^{p-1} e^{-E_{\mathrm{sec}} / \epsilon}}{\epsilon} & \text { for } E_{\mathrm{sec}} \leq 5 \epsilon \\ 0 & \text { for } E_{\mathrm{sec}}>5 \epsilon\end{cases}
$$

The fits are performed simultaneously over all tune shift data at 2.1 and $5.3 \mathrm{GeV}$ shown in Figs. 2 and 3. Table IV compares the optimized parameters to the initial values. The range and accuracy of the tune shift measurements provides high sensitivity to a number of these parameters. For example, the true SEY $\hat{\delta}_{\mathrm{ts}}$ is determined with an accuracy of better than $3 \%$. On the other hand, the tune shifts are relatively insensitive to the peak energy angular dependence parameters $t_{3}$ and $t_{4}$. Those parameters are poorly constrained. Correlations also limit the predictive power of the fit. The correlation matrix for the optimized SEY parameters is shown in Fig. 28. Note that $p$ and $t_{1}$, parameters that characterize the angular dependence and energy distribution of secondaries are highly anticorrelated. The fitted value for the rediffused component of the SEY $\left(P_{1, r}\right)$ is found to be significantly higher than the value obtained in Ref. [22]; however, in view of the high degree of anticorrelation with the elastic yield value $\left(\hat{P}_{1, e}\right)$ (see Fig. 28$)$, which is found to be low, the uncertainty is large.

\section{SIMULATION RESULTS}

The comparison of modeled (using the optimized SEY parameters) and measured tune shift values is shown in Fig. 29 for the $2.1 \mathrm{GeV}$ positron beam and in Fig. 30 for the $5.3 \mathrm{GeV}$ beam. Simulations based on the optimized SEY parameters agree at a level better than $10 \%$ with measurements of tune shifts for all bunches in the train. Note that

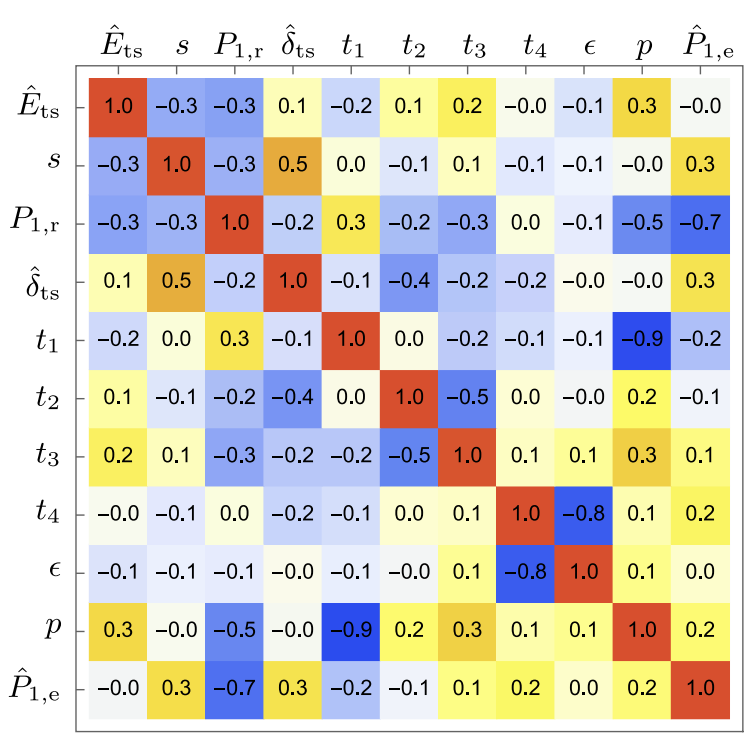

FIG. 28. Correlation matrix for the optimized SEY parameters. Of particular note is that the rediffused yield parameter $P_{1, \mathrm{r}}$ is found to be significantly higher than the initial value, while the elastic yield $\hat{P}_{1, \mathrm{e}}$ is found to be lower, and these two parameters are highly anticorrelated.
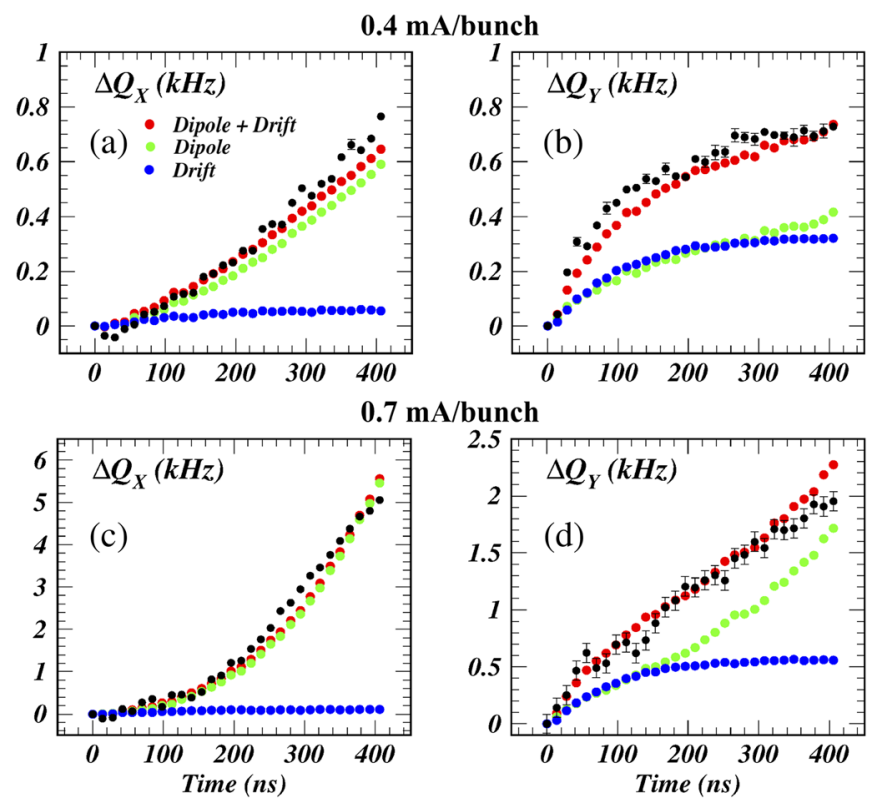

FIG. 29. Comparison of the measured (black points) and modeled tune shift values for the $2.1 \mathrm{GeV}, 30$-bunch train of positrons. The top row shows the tune shift values in the (a) horizontal and (b) vertical planes for a bunch population of $0.64 \times 10^{10}(0.4 \mathrm{~mA} /$ bunch $)$. The bottom row shows the tune shift values in the (c) horizontal and (d) vertical planes for a bunch population of $1.12 \times 10^{10}(0.7 \mathrm{~mA} /$ bunch $)$. Contributions from the field free regions of the ring are shown in blue; those from the dipole regions are shown in green. The sum of the two contributions is shown in red. Bunches are spaced 14 ns apart. 

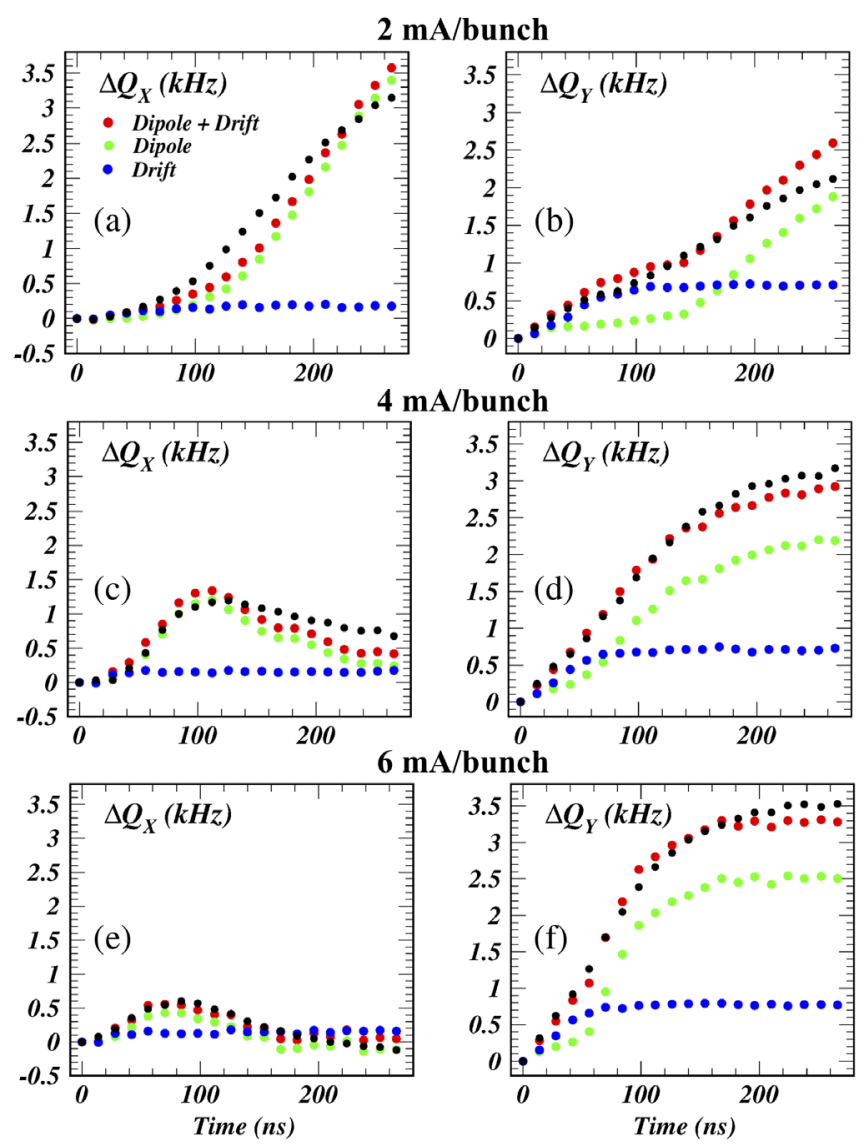

FIG. 30. Comparison of the measured (black points) and modeled tune shift values for the $5.3 \mathrm{GeV}, 20$-bunch train of positrons. The top row shows the tune shift values in the (a) horizontal and (b) vertical planes for a bunch population of $3.2 \times 10^{10}(2 \mathrm{~mA} /$ bunch). The middle row shows the tune shift values in the (c) horizontal and (d) vertical planes for a bunch population of $6.4 \times 10^{10}(4 \mathrm{~mA} /$ bunch $)$. The bottom row shows the tune shift values in the (e) horizontal and (f) vertical planes for a bunch population of $9.6 \times 10^{10}(6 \mathrm{~mA} /$ bunch $)$. Bunches are spaced 14 ns apart.

the tune shifts for different locations along the train and for different beam energy and bunch current are in general dependent on distinct phenomena. For example, the horizontal tune shifts increase by about a factor of 7 when the bunch current is increased from 0.4 to $0.7 \mathrm{~mA} /$ bunch at $2.1 \mathrm{GeV}$. The model shows this dramatic effect to be dominated by a cloud in the dipole sections of the ring. On the other hand, the dipole and field-free regions contribute comparably to the vertical tune shift at $0.4 \mathrm{~mA} /$ bunch and for the first ten bunches of the train at $0.7 \mathrm{~mA} /$ bunch. While the vertical tune shifts saturate at approximately $0.7 \mathrm{kHz}$ for a bunch current of $0.4 \mathrm{~mA} /$ bunch, the dipole regions determine an approximately linear rise during the final 20 bunches at $0.7 \mathrm{~mA} /$ bunch, resulting in a final tune shift value about a factor of 3 higher than at $0.4 \mathrm{~mA} /$ bunch.

Despite the $5.3 \mathrm{GeV}$ bunch populations exceeding those in the $2.1 \mathrm{GeV}$ measurements by nearly a factor of 10 , the vertical tune shifts are less than a factor of 2 higher than those at $2.1 \mathrm{GeV}$, a suppression which cannot be accounted for solely by the beam stiffness. The dipole contributions show a threshold behavior at $2 \mathrm{~mA} /$ bunch similar to that observed at $0.7 \mathrm{~mA} /$ bunch for the $2.1 \mathrm{GeV}$ beam. The contribution of the field-free regions saturates at a level of about $0.5-0.8 \mathrm{kHz}$ at $5.3 \mathrm{GeV}$, roughly independently of bunch current and similar to the level calculated by the model at $2.1 \mathrm{GeV}$ for a bunch current of $0.7 \mathrm{~mA} /$ bunch. At the higher bunch currents, the vertical tune shifts begin to show some saturation, which is attributed to the cloud behavior in the dipole regions of the ring. This saturation, or reduction in tune shift increase, is particularly pronounced in the horizontal tune shifts at $5.3 \mathrm{GeV}$, where again the dominant contributions are from the dipole regions. In fact, the evolution in cloud shape along the train results in increased suppression of the horizontal tune shifts for the higher bunch currents, resulting in a decrease from a level of $3.5 \mathrm{kHz}$, similar to that measured in the vertical plane, to a value less than $0.5 \mathrm{kHz}$ at $6 \mathrm{~mA} /$ bunch.

The validated model was employed to predict tune shifts for future light source operation at $6 \mathrm{GeV}$ at the design beam current of $200 \mathrm{~mA}$. The new combined-function magnet regions and the compact permanent magnet undulator regions were also included in this study. While their ring occupancy fractions are low $3.7 \%$ and $2.9 \%$, respectively), the linear density per positron of absorbed photons can be quite high, owing to the strong magnetic fields and
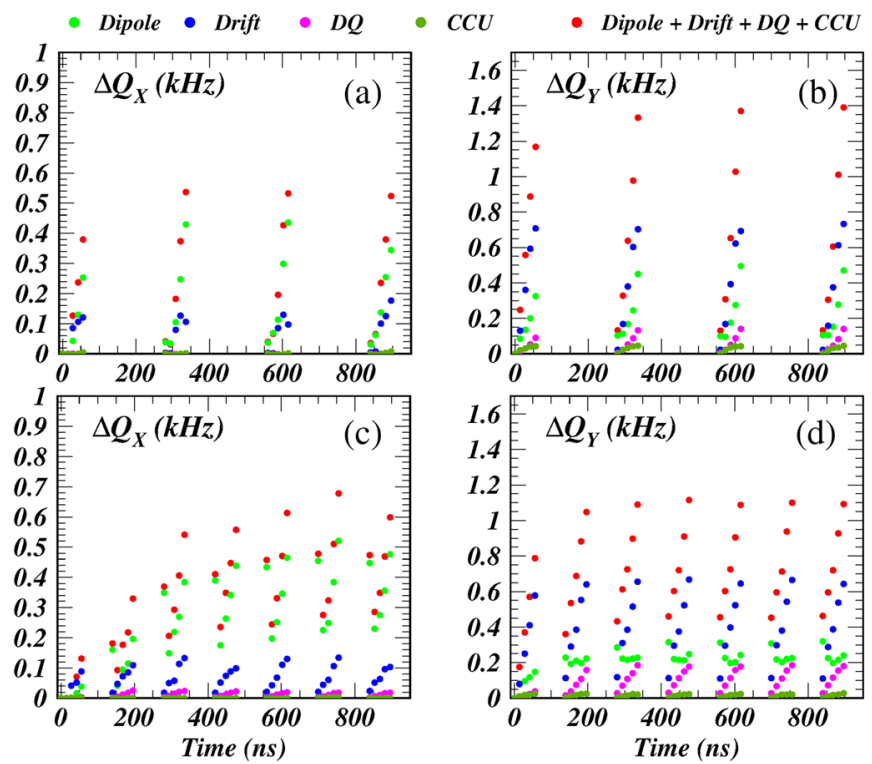

FIG. 31. Modeled tune shifts for the $6.0 \mathrm{GeV}$ CESR upgrade. The upper row shows the tune shifts in the (a) horizontal and (b) vertical planes for the case of nine trains of five bunches with bunch population $7.1 \times 10^{10}$. The lower row shows the tune shifts in the (c) horizontal and (d) vertical planes for the case of 18 trains of five bunches with bunch population $3.5 \times 10^{10}$. The bunch spacing within trains is 14 ns and the trains are equally spaced around the ring. 
the locations of the magnets in the lattice. Nonetheless, the contributions from these new magnets were found to be small, as shown in Fig. 31.

We considered two configurations of five-bunch trains equally spaced throughout the ring giving the design value of $200 \mathrm{~mA}$ for the total beam current. For 18 trains, or nine trains with twice the bunch population, the simulations show that the tune shifts reach an equilibrium value following the passage of just a few trains, which will be the level reached for the stored beam. The maximum tune shift along the train is found to be less than $2 \mathrm{kHz}$. While the dipole regions provide the largest contribution to the horizontal tune shifts, the vertical tune shifts show the fieldfree regions to dominate, owing to the short trains. Since our measurements and modeling of tune shifts in the pre2019 high-current light source operation indicated that stable operation was maintained with tune shifts of about $3 \mathrm{kHz}$, we conclude that the tune shifts from electron cloud buildup will not prohibit reliable operation with positrons at the upgraded light source for the design beam current of $200 \mathrm{~mA}$.

\section{SUMMARY}

We have obtained improved measurements of coherent betatron tune shifts along trains of positron bunches in the horizontal and vertical planes for bunch populations ranging from $0.64 \times 10^{10}$ to $9.6 \times 10^{10}$ at 2.1 and $5.3 \mathrm{GeV}$, enabling advances in the predictive power of electron cloud buildup modeling. Numerical simulation codes for photon tracking and photoelectron production using a detailed model of the storage ring vacuum chamber were employed to eliminate the ad hoc assumptions in electron production rates and kinematics endemic to prior buildup simulations. A parametric model for secondary-yield processes was used in the electron cloud buildup simulation to determine optimized parameters by fitting the modeled tune shift values to the those measured. Excellent agreement with the measurements was obtained for a wide variety of tune shift patterns along the train, allowing conclusions relating the tune shifts to various cloud buildup characteristics. The model was then employed to predict the magnitude of tune shifts expected during future operation of the Cornell Electron-Positron Storage ring as a high-brightness $6 \mathrm{GeV}$ positron light source. This study provides a high degree of confidence that stable operation at $200 \mathrm{~mA}$ beam current can be achieved with either nine or 18 trains of five positron bunches each. The generality and modularity of this modeling procedure addresses the goal of the CESR Test Accelerator program to provide design and diagnostic tools to other present and future accelerator facilities.

\section{ACKNOWLEDGMENTS}

The authors wish to acknowledge important contributions from the technical staffs of the Wilson Laboratory.
We thank Robert Meller for the design and implementation of the tune tracker for betatron tune measurements. This work is supported by the National Science Foundation under contracts No. PHY-0734867, No. PHY-1002467, and by the U.S. Department of Energy under contracts No. DEFC02-08ER41538 and No. DE-SC0006505.

[1] K. Ohmi, S. Heifets, and F. Zimmermann, Study of coherent tune shift caused by electron cloud in positron storage rings, in Proceedings of the Second Asian Particle Accelerator Conference, Beijing, 2001 (Institute of High Energy Physics, Beijing, 2001), pp. 445-447, http:// accelconf.web.cern.ch/AccelConf/a01/PDF/WEP056.pdf.

[2] G. Rumolo, A. Z. Ghalam, T. Katsouleas, C. K. Huang, V. K. Decyk, C. Ren, W. B. Mori, F. Zimmermann, and F. Ruggiero, Electron cloud effects on beam evolution in a circular accelerator, Phys. Rev. ST Accel. Beams 6, 081002 (2003).

[3] M. Billing, The conversion of CESR to operate as the Test Accelerator, CESRTA. Part 1: overview, J. Instrum. 10, P07012 (2015).

[4] The CESR Test Accelerator Electron Cloud Research Program Phase I Report, edited by M. A. Palmer, M. G. Billing, G. F. Dugan, M. A. Furman, and D. L. Rubin (LEPP, Cornell University, Ithaca, NY, 2013), https://www .classe.cornell.edu/public/CLNS/2012/CLNS12-2084/.

[5] J. A. Crittenden, J. R. Calvey, G. F. Dugan, D. L. Kreinick, Z. Leong, J. A. Livezey, M. A. Palmer, D. L. Rubin, D. C. Sagan, K. Harkay, R. L. Holtzapple, M. A. Furman, G. Penn, M. Venturini, M. Pivi, and L. Wang, Progress in studies of electron-cloud-induced optics distortions at CESRTA, in Proceedings of the International Particle Accelerator Conference, Kyoto, Japan (ICR, Kyoto, 2010), pp. 1976-1978, http://accelconf.web.cern.ch/AccelConf/ IPAC10/papers/tupd024.pdf.

[6] D. L. Kreinick, J. A. Crittenden, G. Dugan, M. A. Palmer, G. Ramirez, R. L. Holtzapple, M. Randazzo, M. A. Furman, and M. Venturini, Application of coherent tune shift measurements to the characterization of electron cloud growth, in Proceedings of the 2011 Particle Accelerator Conference, New York, NY (IEEE, New York, 2011), pp. 1680-1682, http://accelconf.web.cern.ch/AccelConf/ PAC2011/papers/wep108.pdf.

[7] G. Dugan et al., Observations and predictions at CESRTA, and outlook for ILC, in Proceedings of ECLOUD 2012: Joint INFN-CERN-EuCARD-AccNet Workshop on Electron-Cloud Effects, La Biodola, Elba, Italy, edited by R. Cimino, G. Rumolo, and F. Zimmermann (CERN, Geneva, Switzerland, 2013), pp. 31-41, http://cds.cern.ch/record/ 1605053.

[8] J. Crittenden, Y. Li, S. Poprocki, and J. S. Soucie, Electron cloud simulations for the low-emittance upgrade at the cornell electron storage ring, in NAPAC2016: Proceedings of the North American Particle Accelerator Conference, Chicago, IL, Report No. TUPOB23, 2016, http://vrws.de/napac2016/ papers/tupob23.pdf.

[9] S. Poprocki, J. Crittenden, S. Hearth, J. Perrin, D. L. Rubin, and S. Wang, Incoherent vertical emittance growth from electron cloud at CESRTA, in IPAC2016: Proceedings of the 
7th International Particle Accelerator Conference, Busan, Korea, Report No. TUPOR021, 2016, http://accelconf.web .cern.ch/AccelConf/ipac2016/papers/tupor021.pdf.

[10] S. Poprocki, J. Crittenden, S. Hearth, J. Perrin, D. Rubin, and S. Wang, Incoherent vertical emittance growth from electron cloud at CESRTA, in NAPAC2016: Proceedings of the North American Particle Accelerator Conference, Chicago, IL, Report No. WEA2CO03, 2016, http://vrws .de/napac2016/papers/tupob23.pdf.

[11] G. Dugan and D. Sagan, Simulating synchrotron radiation in accelerators including diffuse and specular reflections, Phys. Rev. Accel. Beams 20, 020708 (2017).

[12] S. Agostinelli et al., Geant4-a simulation toolkit, Nucl. Instrum. Methods Phys. Res., Sect. A 506, 250 (2003).

[13] S. Buechele, Modeling of electron cloud in CHESS and CHESS-U, NSF REU Report, 2017, https://cornell.app .box.com/s/qepb4oec262bow66p8m6kaei1ct3nfbw.

[14] J. Shanks, J. Barley, S. Barrett, M. Billing, G. Codner, Y. Li, X. Liu, A. Lyndaker, D. Rice, N. Rider, D. L. Rubin, A. Temnykh, and S. T. Wang, Accelerator design for the Cornell High Energy Synchrotron Source upgrade, Phys. Rev. Accel. Beams 22, 021602 (2019).

[15] R. E. Meller and M. A. Palmer, Digital tune tracker for CESR, in Proceedings of the 2011 Particle Accelerator Conference, New York, NY (IEEE, New York, 2011), pp. 504-506, http://accelconf.web.cern.ch/AccelConf/ PAC2011/papers/mop215.pdf.

[16] H. Bruining, Physics and Applications of Secondary Electron Emission (Pergamon Press, London, 1954), https://archive.org/details/SecondaryElectronEmission/ page/n3.

[17] J. Allison et al., Recent developments in Geant4, Nucl. Instrum. Methods Phys. Res., Sect. A 835, 186 (2016).

[18] J. Apostolakis et al., Progress in Geant4 electromagnetic physics modelling and validation, J. Phys. Conf. Ser. 664, 072021 (2015).

[19] G. Rumolo and F. Zimmermann, Practical User Guide for ECloud (CERN, Geneva, Switzerland, 2002), http:// cdsweb.cern.ch/record/702697.

[20] J. A. Crittenden and J. P. Sikora, Electron cloud buildup characterization using shielded pickup measurements and custom modeling code at CESRTA, in Proceedings of ECLOUD 2012: Joint INFN-CERN-EuCARD-AccNet Workshop on Electron-Cloud Effects, La Biodola, Elba, Italy, edited by R. Cimino, G. Rumolo, and F. Zimmermann (CERN, Geneva, Switzerland, 2013), pp. 241-250, http:// cds.cern.ch/record/1562274.

[21] D. L. Kreinick, J. A. Crittenden, G. Dugan, Z. Leong, M. A. Palmer, R. L. Holtzapple, M. Randazzo, M. A. Furman, and M. Venturini, Using coherent tune shifts to evaluate electron cloud effects on beam dynamics at CESRTA, in Proceedings of ECLOUD 2010: 49th ICFA Advanced Beam Dynamics Workshop on Electron Cloud Physics, Ithaca, NY, edited by K. Smolenski (Cornell University, Ithaca, NY, 2013), pp. 130-133, http:// accelconf.web.cern.ch/AccelConf/ECLOUD2010/papers/ PST10.pdf.

[22] M. A. Furman and M. T. F. Pivi, Probabilistic model for the simulation of secondary electron emission, Phys. Rev. ST Accel. Beams 5, 124404 (2002).
[23] D. Sagan, Bmad: A relativistic charged particle simulation library, Nucl. Instrum. Methods Phys. Res., Sect. A 558, 356 (2006).

[24] B. L. Henke, E. M. Gullikson, and J. C. Davis, X-Ray Interactions: Photoabsorption, Scattering, Transmission, and Reflection at $E=50-30,000 \mathrm{eV}, \quad Z=1-92$, At. Data Nucl. Data Tables 54, 181 (1993).

[25] G. F. Dugan, K. G. Sonnad, R. Cimino, T. Ishibashi, and F. Schäfers, Measurements of x-ray scattering from accelerator vacuum chamber surfaces, and comparison with an analytical model, Phys. Rev. ST Accel. Beams 18, 040704 (2015).

[26] V. Ivanchenko, Electromagnetic physics, CERN GEANT4 Web, 2013, https://twiki.cern.ch/twiki/bin/view/Geant4/ ElectromagneticPhysics.

[27] S. Incerti, Journal publications on electromagnetic physics, CERN GEANT4 Web, 2012, https://twiki.cern.ch/twiki/bin/ view/Geant4/EMJournalPublications.

[28] J. Apostolakis, S. Giani, M. G. Pia, U. L., M. Maire, and P. Nieminen, GEANT4 low-energy electromagnetic models for electrons and photons, Report No. CERN-OPEN-99034, 1999, https://cds.cern.ch/record/397049.

[29] G. Cirrone, G. Cuttone, F. D. Rosa, L. Pandola, F. Romano, and Q. Zhang, Validation of the Geant4 electromagnetic photon cross-sections for elements and compounds, Nucl. Instrum. Methods Phys. Res., Sect. A 618, 315 (2010).

[30] J. Apostolakis, A. Bagulya, S. Elles, V. N. Ivanchenko, J. Jacquemier, M. Maire, T. Toshito, and L. Urban, Validation and verification of Geant4 standard electromagnetic physics, J. Phys. Conf. Ser. 219, 032044 (2010).

[31] S. Chauvie, S. Guatelli, V. Ivanchenko, F. Longo, A. Mantero, B. Mascialino, P. Nieminen, L. Pandola, S. Parlati, L. Peralta, M. G. Pia, M. Piergentili, P. Rodrigues, S. Saliceti, and A. Tnndade, Geant4 low energy electromagnetic physics, in IEEE Symposium Conference Record Nuclear Science 2004 (IEEE, 2004), Vol. 3, pp. 18811885, https://ieeexplore.iee.org/document/1462612.

[32] V. Ivanchenko, J. Apostolakis, A. Bagulya, H. Ben Abdelouahed, R. Black, A. Bogdanov, H. Burkhard, S. Chauvie, P. Cirrone, G. Cuttone, G. Depaola, F. Rosa, S. Elles, Z. Francis, V. Grichine, P. Gumplinger, P. Gueye, S. Incerti, A. Ivanchenko, and C. Zacharatou, Recent Improvements in Geant4 Electromagnetic Physics Models and Interfaces, Prog. Nucl. Sci. Technol. 2, 898 (2011).

[33] S. Guatelli, A. Mantero, B. Mascialino, P. Nieminen, and M. Pia, Geant4 atomic relaxation, IEEE Trans. Nucl. Sci. 54, 585 (2007).

[34] M. G. Billing, J. Conway, E. E. Cowan, J. A. Crittenden, W. Hartung, J. Lanzoni, Y. Li, C. S. Shill, J. P. Sikora, and K. G. Sonnad, Measurement of electron trapping in the Cornell Electron Storage Ring, Phys. Rev. ST Accel. Beams 18, 041001 (2015).

[35] J. S. Berg, Energy gain in an electron cloud during the passage of a bunch, Report No. LHC-Project-Note-97, 1997, https://cds.cern.ch/record/692004.

[36] K. Rowan, Revised Predictions of Electron-CloudBased Instabilities for CHESS-U, NSF REU Report, 2018, https://cornell.app.box.com/s/n0fh73ejawiwp9ngl4ndrce754ltdmmn. 
[37] F. Zimmermann, G. Rumolo, and K. Ohmi, Electron cloud build up in machines with short bunches, in ICFA Beam Dynamics Newsletter, No. 33, edited by K. Ohmi and M. Furman (International Committee on Future Accelerators, 2004), pp. 14-24, https://icfa-usa.jlab.org/archive/ newsletter/icfa_bd_nl_33.pdf.

[38] J. Calvey, M. Billing, W. Hartung, J. Perrin, D. Rubin, D. Sagan, and S. T. Wang, Measurement and modeling of single bunch wake field effects in CESR, in IPAC2015: Proceedings of the 6th International Particle Accelerator Conference, Richmond, VA, 2015, Report No. MOPMA056, https://jacowfs.jlab.org/conf/y15/ ipac15/prepress/MOPMA056.PDF.

[39] F. Le Pimpec, R. E. Kirby, F. K. King, and M. Pivi, Electron conditioning of technical aluminium surfaces:
Effect on the secondary electron yield, J. Vac. Sci. Technol. A 23, 1610 (2005).

[40] F. Le Pimpec, F. King, and R. E. Kirby, Electron Conditioning of Technical Aluminum Surfaces (Linear Collider Collaboration/SLAC, Stanford, CA, 2004) (Tech. Rep. LCC-0153/SLAC-TN-04-051), http://www-project .slac.stanford.edu/lc/ilc/TechNotes/LCCNotes/PDF/LCC0153.pdf

[41] V. Baglin, J. Bojko, O. Gröbner, B. Henrist, N. Hilleret, C. Scheuerlein, and M. Taborelli, The secondary electron yield of technical materials and its variation with surface treatments, in Proceedings of the European Particle Accelerator Conference, Vienna, 2000 (EPS, Geneva, 2000), pp. 217-221, http://accelconf.web.cern.ch/AccelConf/e00/ PAPERS/THXF102.pdf. 